\title{
Representability conditions by Grassmann integration
}

\author{
Volker Bach, Hans Konrad Knörr and Edmund Menge
}

\begin{abstract}
Representability conditions on the one- and two-particle density matrix for fermion systems are formulated by means of Grassmann integrals. A positivity condition for a certain kind of Grassmann integral is established which by an appropriate choice of the integrand, in turn, induces the well-known G-, P- and Q-Conditions of quantum chemistry. Similarly, the $\mathrm{T}_{1^{-}}$and $\mathrm{T}_{2}$-Conditions are derived. Furthermore, quasifree Grassmann states are introduced and, for every operator $\widetilde{\gamma} \in \mathcal{H} \oplus \mathcal{H}$ with $0 \leq \widetilde{\gamma} \leq \mathbb{1}$, the existence of a unique quasifree Grassmann state whose one-particle density matrix is $\widetilde{\gamma}$ is shown.
\end{abstract}

1 Introduction

2 Reduced density matrices and representability

3 Grassmann algebras

4 Grassmann integration

5 Representability conditions from Grassmann integrals

5.1 Conditions on the one-particle density matrix

5.2 G-, P-, and Q-Condition

5.3 $\mathrm{T}_{1}$ - and generalized $\mathrm{T}_{2}$-Condition

6 Quasifree Grassmann states

References 


\section{Introduction}

The grand canonical energy (minus pressure) $E_{0}(\mu):=\inf \{\sigma\{\widehat{\mathbb{H}}-\mu \widehat{\mathbb{N}}\}\}$ at sufficiently large chemical potential $\mu \geq 0$ of a quantum system with a Hamiltonian $\widehat{\mathbb{H}}$ and particle number operator $\widehat{\mathbb{N}}$ is given by the RayleighRitz principle as

$$
E_{0}(\mu)=\inf \left\{\operatorname{tr}\left(\rho^{\frac{1}{2}}(\widehat{\mathbb{H}}-\mu \widehat{\mathbb{N}}) \rho^{\frac{1}{2}}\right) \mid \rho \in D M\right\},
$$

where $\widehat{\mathbb{H}}=\widehat{\mathbb{H}}^{*}$ is a self-adjoint operator obeying stability of matter, i. e., which is bounded below by $-c \widehat{\mathbb{N}}$ for some $c<\infty$ and at most quartic in the creation and annihilation operators $[11,18]$. This is typically the case for models of non-relativistic matter in physics and chemistry. The Pauli principle plays a crucial role for stability of matter to hold true and we, thus, restrict our attention to fermion systems. On the fermion Fock space $\wedge \mathcal{H}$ the variation on the r. h.s. of Eq. (1.1) is over the set

$$
D M:=\left\{\rho \mid \rho \in \mathcal{L}_{+}^{1}(\wedge \mathcal{H}), \operatorname{tr}(\rho)=1,\left\langle\widehat{\mathbb{N}}^{2}\right\rangle_{\rho}<\infty\right\},
$$

i. e., density matrices with finite particle number variance. Here, the expectation value of an observable $\widehat{\mathbb{A}}$ is

$$
\langle\widehat{\mathbb{A}}\rangle_{\rho}:=\operatorname{tr}\left(\rho^{\frac{1}{2}} \widehat{\mathbb{A}} \rho^{\frac{1}{2}}\right) .
$$

More specifically, if

$$
\widehat{\mathbb{H}}-\mu \widehat{\mathbb{N}}=\sum_{k, m} h_{k m} c^{*}\left(f_{k}\right) c\left(f_{m}\right)+\sum_{k, l, m, n} V_{k l m n} c^{*}\left(f_{l}\right) c^{*}\left(f_{k}\right) c\left(f_{m}\right) c\left(f_{n}\right),
$$

then

$$
E_{0}(\mu)=\inf \left\{\mathcal{E}\left(\gamma_{\rho}, \Gamma_{\rho}\right) \mid \rho \in D M\right\}
$$

where

$$
\mathcal{E}\left(\gamma_{\rho}, \Gamma_{\rho}\right):=\sum_{k, m} h_{k m}\left\langle f_{m}, \gamma_{\rho} f_{k}\right\rangle+\sum_{k, l, m, n} V_{k l m n}\left\langle f_{m} \otimes f_{n}, \Gamma_{\rho}\left(f_{k} \otimes f_{l}\right)\right\rangle .
$$


The one- and two-particle density matrices corresponding to $\rho$ are defined by

$$
\begin{aligned}
\left\langle f, \gamma_{\rho} g\right\rangle & :=\left\langle c^{*}(g) c(f)\right\rangle_{\rho} \quad \text { and } \\
\left\langle f \otimes g, \Gamma_{\rho}(\tilde{f} \otimes \tilde{g})\right\rangle & :=\left\langle c^{*}(\tilde{g}) c^{*}(\tilde{f}) c(f) c(g)\right\rangle_{\rho}
\end{aligned}
$$

respectively, for all $f, g, \tilde{f}, \tilde{g} \in \mathcal{H}$. Note that (1.2) can be rewritten as

$$
E_{0}(\mu)=\inf \{\mathcal{E}(\gamma, \Gamma) \mid(\gamma, \Gamma) \in \mathcal{R}\}
$$

where

$$
\mathcal{R}:=\left\{(\gamma, \Gamma) \in \mathcal{L}^{1}(\mathcal{H}) \times \mathcal{L}^{1}(\mathcal{H} \otimes \mathcal{H}) \mid \exists \rho \in D M:(\gamma, \Gamma)=\left(\gamma_{\rho}, \Gamma_{\rho}\right)\right\}
$$

denotes the set of all representable one- and two-particle density matrices. Eq. (1.3) suggests that the search for a minimizing $\rho$ could be drastically simplified if one would find a characterization of all representable reduced density matrices $(\gamma, \Gamma)$. This was realized almost fifty years ago $[5,7,9,12]$, but such a characterization is still unknown.

The characterization of $E_{0}(\mu)$ by the variation (1.3) immediately yields lower bounds of the form

$$
E_{0}(\mu)=: E_{\mathcal{R}}(\mu) \geq E_{\mathcal{S}}(\mu)
$$

for any superset $\mathcal{S}$ of $\mathcal{R}$. For example, the positivity $\left\langle P_{2}^{*} P_{2}\right\rangle_{\rho} \geq 0$ for all polynomials $P_{2} \equiv P_{2}\left(c^{*}, c\right)$ in the creation and annihilation operators of degree two yields the so-called G-, P-, and Q-Conditions on $\left(\gamma_{\rho}, \Gamma_{\rho}\right)[2,5,7,9]$. Similarly, the positivity $\left\langle P_{3}^{*} P_{3}+P_{3} P_{3}^{*}\right\rangle_{\rho} \geq 0$ yields the $\mathrm{T}_{1^{-}}$and generalized $\mathrm{T}_{2}$-Conditions [7]. Hence, all representable reduced density matrices $(\gamma, \Gamma)$ necessarily fulfill the $\mathrm{G}_{-}, \mathrm{P}_{-}, \mathrm{Q}_{-}, \mathrm{T}_{1^{-}}$, and generalized $\mathrm{T}_{2}$-Conditions, and we have

$$
E_{\mathcal{R}}(\mu) \geq E_{\mathcal{S}\left[\mathrm{G}, \mathrm{P}, \mathrm{Q}, \mathrm{T}_{1}, \mathrm{~T}_{2}\right]}(\mu) \geq E_{\mathcal{S}[\mathrm{G}, \mathrm{P}, \mathrm{Q}]}(\mu)
$$

since $\mathcal{R} \subseteq \mathcal{S}\left[\mathrm{G}, \mathrm{P}, \mathrm{Q}, \mathrm{T}_{1}, \mathrm{~T}_{2}\right] \subseteq \mathcal{S}[\mathrm{G}, \mathrm{P}, \mathrm{Q}]$, with

$$
\mathcal{S}[X]:=\left\{(\gamma, \Gamma) \in \mathcal{L}^{1}(\mathcal{H}) \times \mathcal{L}^{1}(\mathcal{H} \otimes \mathcal{H}) \mid(\gamma, \Gamma) \text { fulfills Condition } X\right\} .
$$

We have discussed (1.4) and (1.5) for $\mathcal{S}=\mathcal{S}[\mathrm{G}, \mathrm{P}]$ in some detail in [2] and refer the reader to that paper and references therein. Furthermore, for 
$\mathcal{S}=\mathcal{S}\left[\mathrm{G}, \mathrm{P}, \mathrm{Q}, \mathrm{T}_{1}, \mathrm{~T}_{2}\right]$ numerical works show agreement with Full CI computations $[4,13,14,19]$ to high accuracy.

The purpose of the present paper is the reformulation of representability conditions in terms of Grassmann integrals. Such a transcription may possibly yield new viewpoints and hopefully new insights into the representability problem. To this end, we introduce a Grassmann algebra $\mathcal{G}_{M}$ as a finite dimensional complex algebra. The object on $\mathcal{G}_{M}$ corresponding to a given density matrix is an element of the form $\vartheta^{*} \star \vartheta$ described in the sequel. Grassmann integration is the basic and most commonly used method (see, e.g., $[8,16]$ ) in theoretical physics to compute partition functions of the form

$$
Z_{\Gamma, \lambda}(J):=\int \mathrm{D}_{\Gamma}(\phi) \mathrm{e}^{-S_{\Gamma}+(J, \phi)_{\Gamma}}
$$

as a functional integral with $\mathrm{D}_{\Gamma}(\phi):=\prod_{x \in \Gamma} \mathrm{d} \phi(x)$ with sources $J: \Gamma \rightarrow \mathbb{R}$ and an action $S_{\Gamma}$ (see [16] for further details).

The derivation of the G-, P-, Q-, $\mathrm{T}_{1^{-}}$, and generalized $\mathrm{T}_{2}$-Conditions is based on the representation of the trace on $\wedge \mathcal{H}$ in terms of Grassmann integrals and a positivity condition of a Grassmann integral, namely

$$
\forall \eta \in \mathcal{G}_{M}: \int \mathrm{d}(\bar{\Psi}, \Psi) \mathrm{e}^{2(\bar{\Psi}, \Psi)} \eta^{*} \star \eta \geq 0,
$$

where $\int \mathrm{d}(\bar{\Psi}, \Psi)$ denotes the Grassmann integration. The star product refers to a product on $\mathcal{G}_{M}$ and is introduced later. Considering appropriate subspaces of $\mathcal{G}_{M}$ denoted by $\mathcal{G}_{M}^{(n)}$, the main results of this paper are the bounds for the one-particle density matrix $\gamma_{\vartheta}$,

$$
\left\{\forall \mu \in \mathcal{G}_{M}^{(1)}: \int \mathrm{d}(\bar{\Psi}, \Psi) \mathrm{e}^{2(\bar{\Psi}, \Psi)} \vartheta^{*} \star \vartheta \star \mu \geq 0\right\} \quad \Leftrightarrow \quad\left\{0 \leq \gamma_{\vartheta} \leq \mathbb{1}\right\},
$$

and the G-, P-, and Q-Condition as conditions for the two-particle density matrix $\Gamma_{\vartheta}$,

$$
\begin{aligned}
& \left\{\forall \mu \in \mathcal{G}_{M}^{(2)}: \int \mathrm{d}(\bar{\Psi}, \Psi) \mathrm{e}^{2(\bar{\Psi}, \Psi)} \vartheta^{*} \star \vartheta \star \mu \geq 0\right\} \\
& \Leftrightarrow \quad\left\{0 \leq \gamma_{\vartheta} \leq \mathbb{1}, \text { G- }, \text { P-, and Q-Condition }\right\} .
\end{aligned}
$$


Finally, we prove the validity of the $\mathrm{T}_{1^{-}}$and generalized $\mathrm{T}_{2}$-Condition deduced from Ineq. (1.6).

\section{Reduced density matrices and representability}

Before we elucidate how to derive the $\mathrm{G}_{-}^{-}, \mathrm{P}_{-}, \mathrm{Q}_{-}, \mathrm{T}_{1^{-}}$, and generalized $\mathrm{T}_{2^{-}}$ Conditions for the 1- and 2-particle density matrix (1- and 2-pdm) by Grassmann integration, we give a definition of these first two reduced density matrices. For this purpose, we consider a finite-dimensional index set $M$, an $|M|$-dimensional (one-particle) Hilbert space $(\mathcal{H},\langle\cdot, \cdot\rangle)$, and an arbitrary, but fixed orthonormal basis (ONB) $\left\{\psi_{i}\right\}_{i \in M}$ of $\mathcal{H}$. Furthermore, we introduce the usual fermion creation and annihilation operators on the fermion Fock space $\wedge \mathcal{H}$ over $\mathcal{H}$ given by $c^{*}\left(\psi_{i}\right) \equiv c_{i}^{*}$ and $c\left(\psi_{i}\right) \equiv c_{i}$ with the canonical anticommutation relations $(\mathrm{CAR})$

$$
\{c(f), c(g)\}=\left\{c^{*}(f), c^{*}(g)\right\}=0 \text { and }\left\{c(f), c^{*}(g)\right\}=\langle f, g\rangle \cdot \mathbb{1}
$$

for all $f, g \in \mathcal{H}$, where $\langle\cdot, \cdot\rangle$ is linear in the second and antilinear in the first argument. $\{A, B\}:=A B+B A$ denotes the anticommutator.

The 1-pdm $\gamma_{\rho} \in \mathcal{L}_{+}^{1}(\mathcal{H})$ of a density matrix $\rho$, i. e., a positive trace class operator on $\wedge \mathcal{H}$ of unit trace $\left(\operatorname{tr}_{\wedge \mathcal{H}}(\rho)=1\right)$, is defined by its matrix elements as

$$
\forall f, g \in \mathcal{H}:\left\langle f, \gamma_{\rho} g\right\rangle:=\operatorname{tr}_{\wedge \mathcal{H}}\left(\rho c^{*}(g) c(f)\right)
$$

Likewise, the 2 -pdm $\Gamma_{\rho} \in \mathcal{L}_{+}^{1}(\mathcal{H} \otimes \mathcal{H})$ of $\rho$ is defined by

$$
\begin{aligned}
& \forall f_{1}, f_{2}, g_{1}, g_{2} \in \mathcal{H}: \\
& \left\langle f_{1} \otimes f_{2}, \Gamma_{\rho}\left(g_{1} \otimes g_{2}\right)\right\rangle:=\operatorname{tr}_{\wedge \mathcal{H}}\left(\rho c^{*}\left(g_{2}\right) c^{*}\left(g_{1}\right) c\left(f_{1}\right) c\left(f_{2}\right)\right) .
\end{aligned}
$$

There are several properties which can be derived directly from the definition of $\gamma_{\rho}$ and $\Gamma_{\rho}$.

Lemma 2.1. Let $\rho \in \mathcal{L}_{+}^{1}(\wedge \mathcal{H})$ be a density matrix and $\widehat{\mathbb{N}}:=\sum_{k \in M} c_{k}^{*} c_{k}$ the particle number operator with $\left\langle\widehat{\mathbb{N}}^{2}\right\rangle_{\rho}<\infty$. Then the following assertions hold true:

(i) $\gamma_{\rho} \in \mathcal{L}_{+}^{1}(\mathcal{H}), 0 \leq \gamma_{\rho} \leq \mathbb{1}, \operatorname{tr}_{\mathcal{H}}\left(\gamma_{\rho}\right)=\langle\widehat{\mathbb{N}}\rangle_{\rho}, \Gamma_{\rho} \in \mathcal{L}_{+}^{1}(\mathcal{H} \otimes \mathcal{H}), 0 \leq \Gamma_{\rho} \leq$ $\langle\widehat{\mathbb{N}}\rangle_{\rho} \cdot \mathbb{1}$, and $\operatorname{tr}_{\mathcal{H} \otimes \mathcal{H}}\left(\Gamma_{\rho}\right)=\langle\widehat{\mathbb{N}}(\widehat{\mathbb{N}}-\mathbb{1})\rangle_{\rho}$. 
(ii) If $\operatorname{Ran}(\rho) \subseteq \wedge^{(N)} \mathcal{H}, N \in \mathbb{N}$, then, for all $f, g \in \mathcal{H}$,

$$
\left\langle f, \gamma_{\rho} g\right\rangle=\frac{1}{N-1} \sum_{k \in M}\left\langle f \otimes \psi_{k}, \Gamma_{\rho}\left(g \otimes \psi_{k}\right)\right\rangle
$$

where $\left\{\psi_{k}\right\}_{k \in M}$ is an ONB of $\mathcal{H}$. Here, $\wedge^{(N)} \mathcal{H}$ denotes the fermion $N$-particle Fock space.

(iii) Furthermore,

$$
\rho=\left|c^{*}\left(\psi_{1}\right) \cdots c^{*}\left(\psi_{N}\right) \Omega\right\rangle\left\langle c^{*}\left(\psi_{1}\right) \cdots c^{*}\left(\psi_{N}\right) \Omega\left|\quad \Leftrightarrow \quad \gamma_{\rho}=\sum_{i=1}^{N}\right| \psi_{i}\right\rangle\left\langle\psi_{i}\right|
$$

and, in this case,

$$
\Gamma_{\rho}=(\mathbb{1}-\operatorname{Ex})\left(\gamma_{\rho} \otimes \gamma_{\rho}\right),
$$

where $\operatorname{Ex}(f \otimes g):=g \otimes f$ for any $f, g \in \mathcal{H}$.

For further details we recommend $[1,2,5,9]$ and a proof can be found in [1]. Beside these properties, necessary conditions on $(\gamma, \Gamma)$ to be representable were derived in $[5,7,9]$. In particular, the $\mathrm{P}-$, G-, and Q-Conditions are

(P) $\{(\gamma, \Gamma)$ fulfills P-Condition $\} \quad: \Leftrightarrow \quad\{\Gamma \geq 0\}$,

(G) $\{(\gamma, \Gamma)$ fulfills G-Condition $\}$

$$
: \Leftrightarrow \quad\left\{\forall A \in \mathcal{B}(\mathcal{H}): \operatorname{tr}\left(\left(A^{*} \otimes A\right)(\Gamma+\operatorname{Ex}(\gamma \otimes \mathbb{1}))\right) \geq|\operatorname{tr}(A \gamma)|^{2}\right\},
$$

(Q) $\quad\{(\gamma, \Gamma)$ fulfills Q-Condition $\}$

$$
: \Leftrightarrow\{\Gamma+(\mathbb{1}-\operatorname{Ex})(\mathbb{1} \otimes \mathbb{1}-\gamma \otimes \mathbb{1}-\mathbb{1} \otimes \gamma) \geq 0\} .
$$

The $\mathrm{T}_{1}$ - and generalized $\mathrm{T}_{2}$-Conditions are more complicated and not given here. For these conditions we refer the reader to [7] or Subsection 5.3 of this work.

\section{Grassmann algebras}

We introduce the Grassmann algebra as the complex algebra generated by elements of the set $\left\{\bar{\psi}_{i}, \psi_{i}\right\}_{i \in M}$ with $|M|<\infty$ modulo the anticommutation relations specified below. A product of two generators is denoted by $\psi_{i} \cdot \psi_{j} \equiv$ $\psi_{i} \psi_{j}$. The unity is given as $1 \cdot \psi_{i}=\psi_{i} \cdot 1=\psi_{i}$ (and equivalently for $\bar{\psi}_{j}$ ). The 
anticommutation relations allow us to find a one-to-one representation of the CAR of fermion creation and annihilation operators in terms of Grassmann variables. For further details on this well-known material we recommend $[6,15-17]$. We use the notation of $[15]$.

Definition 3.1. For an ordered set $I:=\left\{i_{1}, \ldots, i_{m}\right\} \subseteq M$ we write

$$
\Psi_{I}:=\psi_{i_{1}} \cdots \psi_{i_{m}}, \quad \bar{\Psi}_{I}:=\bar{\psi}_{i_{1}} \cdots \bar{\psi}_{i_{m}}
$$

For $I=\emptyset$ we set $\Psi_{I}=\bar{\Psi}_{I}=1$. Denoting the reversely ordered set corresponding to $I$ by $I^{\prime}$, we write

$$
\Psi_{I^{\prime}}:=\psi_{i_{m}} \cdots \psi_{i_{1}}
$$

Definition 3.2. Given a set of generators $\left\{\bar{\psi}_{i}, \psi_{i}\right\}_{i \in M}$ obeying the anticommutation relations

$$
\bar{\psi}_{i} \psi_{j}+\psi_{j} \bar{\psi}_{i}=\bar{\psi}_{i} \bar{\psi}_{j}+\bar{\psi}_{j} \bar{\psi}_{i}=\psi_{i} \psi_{j}+\psi_{j} \psi_{i}=0 \quad \forall i, j \in M
$$

the Grassmann algebra $\mathcal{G}_{M}$ is defined as

$$
\mathcal{G}_{M}:=\operatorname{span}\left\{\bar{\Psi}_{I} \Psi_{J} \mid I, J \subseteq M\right\}
$$

Introducing the ordinary wedge product, we can identify $\mathcal{G}_{M}$ with the Fock space $\wedge(\overline{\mathcal{H}} \oplus \mathcal{H})$ of a Hilbert space $(\mathcal{H},\langle\cdot, \cdot\rangle)$ with finite dimension $|M|$. Considering $\mathcal{H}$ as a subset of $\mathcal{G}_{M}$, we can identify $\left\{\psi_{i}\right\}_{i \in M}$ with a fixed ONB of $\mathcal{H}$ and $\left\{\bar{\psi}_{i}\right\}_{i \in M}$ with the corresponding ONB of $\overline{\mathcal{H}}$, i. e., the space of all continuous linear functionals $\mathcal{H} \rightarrow \mathbb{C}, \psi_{i} \mapsto \bar{\psi}_{i}(\cdot):=\left\langle\psi_{i}, \cdot\right\rangle$.

Remark 3.3. If $\mathcal{G}_{M}$ is generated by $\left\{\bar{\phi}_{i}, \phi_{i}\right\}_{i \in M}$, we emphasize this by using $\mu(\bar{\phi}, \phi) \in \mathcal{G}_{M}$ instead of $\mu \in \mathcal{G}_{M}$. We also use "mixed" generators, e.g.,

$$
\mu(\bar{\psi}, \phi):=\sum_{i, j} \alpha_{i j} \bar{\Psi}_{I_{i}} \Phi_{J_{j}} .
$$

Later, it is necessary to link the CAR algebra of fermion annihilation and creation operators to a Grassmann algebra. For this purpose, a map between $\mathcal{B}(\wedge \mathcal{H})$ and $\mathcal{G}_{M}$ as an isomorphism between vector spaces is required. This map is provided below. 
Definition 3.4. Let $\mathcal{G}_{M}$ be generated by $\left\{\bar{\psi}_{i}, \psi_{i}\right\}_{i \in M}$ and associate $\left\{\psi_{i}\right\}_{i \in M}$ with a fixed ONB of $\mathcal{H}$. For all $z \in \mathbb{C}$ and $\left\{i_{1}, \ldots, i_{m}\right\},\left\{j_{1}, \ldots, j_{n}\right\} \subseteq M$, $m, n \leq|M|$, we define the linear map $\Theta: \mathcal{B}(\wedge \mathcal{H}) \rightarrow \mathcal{G}_{M}$ by $\Theta(z):=z$ and

$$
\Theta\left(c^{*}\left(\psi_{i_{1}}\right) \cdots c^{*}\left(\psi_{i_{m}}\right) c\left(\psi_{j_{1}}\right) \cdots c\left(\psi_{j_{n}}\right)\right):=\bar{\psi}_{i_{1}} \cdots \bar{\psi}_{i_{m}} \psi_{j_{1}} \cdots \psi_{j_{n}},
$$

and extension to $\mathcal{B}(\wedge \mathcal{H})$ by linearity.

We emphasize that $\Theta$ is not multiplicative: While

$$
\Theta\left(c^{*}\left(\psi_{1}\right) c\left(\psi_{1}\right)\right)=\bar{\psi}_{1} \psi_{1}=\Theta\left(c^{*}\left(\psi_{1}\right)\right) \Theta\left(c\left(\psi_{1}\right)\right),
$$

we have

$$
\begin{aligned}
\Theta\left(c\left(\psi_{1}\right) c^{*}\left(\psi_{1}\right)\right) & =\Theta\left(-c^{*}\left(\psi_{1}\right) c\left(\psi_{1}\right)+\mathbb{1}\right) \\
& =-\bar{\psi}_{1} \psi_{1}+1=\psi_{1} \bar{\psi}_{1}+1=\Theta\left(c\left(\psi_{1}\right)\right) \Theta\left(c^{*}\left(\psi_{1}\right)\right)+1
\end{aligned}
$$

Thus, Eq. (3.1) only holds for normal-ordered monomials in creation and annihilation operators, i. e., monomials in which all creation operators are to the left of all annihilation operators.

Definition 3.5. For any $A \in \mathcal{B}(\mathcal{H})$ we set

$$
(\bar{\Psi}, A \Phi):=\sum_{i, j \in M}\left[\bar{\psi}_{i}\left(A \psi_{j}\right)\right] \bar{\psi}_{j} \phi_{i} \in \mathcal{G}_{M} .
$$

Note that $\bar{\psi}_{i}\left(A \psi_{j}\right)=\left\langle\psi_{i}, A \psi_{j}\right\rangle \in \mathbb{C}$. Furthermore, $(\bar{\Psi}, A \Phi)$ does not depend on the choice of generators of $\mathcal{G}_{M}$ as can be seen by a unitary change of generators, e.g., $\chi_{i}:=\sum_{j \in M} U_{i j} \psi_{j}$ for unitary $U$. An important case is $A=\mathbb{1}$. Here we have $(\bar{\Psi}, \Phi)=\sum_{i \in M} \bar{\psi}_{i} \phi_{i}$. One of the last ingredients for the Grassmann integration is the following.

Definition 3.6. The expression $\mathrm{e}^{ \pm(\bar{\Psi}, A \Phi)} \in \mathcal{G}_{M}$ is given by

$$
\mathrm{e}^{ \pm(\bar{\Psi}, A \Phi)}:=\sum_{m=0}^{\infty} \frac{1}{m !}[ \pm(\bar{\Psi}, A \Phi)]^{m} .
$$

As $\operatorname{dim}\{\wedge \mathcal{H}\}=2^{\operatorname{dim} \mathcal{H}}$, the sum runs only over $0 \leq m \leq 2^{\operatorname{dim} \mathcal{H}}$. 
Remark 3.7. Since $(\bar{\Psi}, \Phi)=\sum_{\alpha \in M} \bar{\psi}_{\alpha} \phi_{\alpha}$, and $\bar{\psi}_{\alpha} \phi_{\alpha}$ commutes with every element of $\mathcal{G}_{M}$, we have

$$
\mathrm{e}^{ \pm(\bar{\Psi}, \Phi)}=\prod_{\alpha \in M}\left(1 \pm \bar{\psi}_{\alpha} \phi_{\alpha}\right)
$$

Definition 3.8. For all $i, j \in M$, we define the vector space homomorphisms $\frac{\delta}{\delta \psi_{i}}, \frac{\delta}{\delta \bar{\psi}_{i}}: \mathcal{G}_{M} \rightarrow \mathcal{G}_{M}$ by

$$
\frac{\delta}{\delta \psi_{i}} \psi_{j}=\frac{\delta}{\delta \bar{\psi}_{i}} \bar{\psi}_{j}=\delta_{i j} \quad \text { and } \quad \frac{\delta}{\delta \psi_{i}} \bar{\psi}_{j}=\frac{\delta}{\delta \bar{\psi}_{i}} \psi_{j}=0
$$

Remark 3.9. The set $\left\{\frac{\delta}{\delta \bar{\psi}_{i}}, \frac{\delta}{\delta \psi_{i}}\right\}_{i \in M}$ itself generates a Grassmann algebra.

\section{Grassmann integration}

Now we are prepared to define the Grassmann integral, which is a linear operator from $\mathcal{G}_{M}$ to $\mathbb{C}$.

Definition 4.1. The map $\int \mathrm{d}(\bar{\Psi}, \Psi): \mathcal{G}_{M} \rightarrow \mathbb{C}$ is defined by

$$
\int \mathrm{d}(\bar{\Psi}, \Psi):=\prod_{\alpha \in M}\left(\frac{\delta}{\delta \bar{\psi}_{\alpha}} \frac{\delta}{\delta \psi_{\alpha}}\right)
$$

and is referred to as the Grassmann integral.

Remark 4.2. If the factor $\mathrm{e}^{2(\bar{\Psi}, \Psi)}=\prod_{\alpha \in M}\left(1+2 \bar{\psi}_{\alpha} \psi_{\alpha}\right)$ is involved in the integration, we use the abbreviation

$$
\int \mathcal{D}(\bar{\Psi}, \Psi):=\int \mathrm{d}(\bar{\Psi}, \Psi) \mathrm{e}^{2(\bar{\Psi}, \Psi)}
$$

since $\prod_{\alpha \in M}\left(1+2 \bar{\psi}_{\alpha} \psi_{\alpha}\right)$ commutes with every element of $\mathcal{G}_{M}$.

In order to state the invariance of the Grassmann integration with respect to a change of generators, we introduce some notation. We write two sets of generators, $\left\{\bar{\psi}_{i}, \psi_{i}\right\}_{i \in M}$ and $\left\{\bar{\chi}_{i}, \chi_{i}\right\}_{i \in M}$, as $2|M|$-component vectors 
$\underline{a}$ and $\underline{b}$, respectively, whose entries are given by

$$
a_{i}:=\bar{\psi}_{i} \text { and } a_{|M|+i}:=\psi_{i}, \quad \text { and } \quad b_{i}:=\bar{\chi}_{i} \text { and } b_{|M|+i}:=\chi_{i}
$$

for all $i \in M$. Furthermore, we define the entries of the $2|M|$-component vectors $\frac{\delta}{\delta \underline{a}}$ and $\frac{\delta}{\delta \underline{b}}$ by

$$
\frac{\delta}{\delta \underline{a}_{i}}:=\frac{\delta}{\delta \bar{\psi}_{i}} \text { and } \frac{\delta}{\delta \underline{a}_{|M|+i}}:=\frac{\delta}{\delta \psi_{i}}, \quad \text { and } \quad \frac{\delta}{\delta \underline{b}_{i}}:=\frac{\delta}{\delta \bar{\chi}_{i}} \text { and } \frac{\delta}{\delta \underline{b}_{|M|+i}}:=\frac{\delta}{\delta \chi_{i}} \text {. }
$$

We denote the index set for the introduced vectors by $\widetilde{M},|\widetilde{M}|=2|M|$. In this notation the Grassmann integration with respect to $\left\{\bar{\psi}_{i}, \psi_{i}\right\}_{i \in M}$ reads

$$
(-1)^{\frac{1}{2}|M|(|M|-1)} \prod_{\alpha \in M}\left(\frac{\delta}{\delta \bar{\psi}_{\alpha}} \frac{\delta}{\delta \psi_{\alpha}}\right)=\prod_{\alpha \in M} \frac{\delta}{\delta \bar{\psi}_{\alpha}} \prod_{\alpha \in M} \frac{\delta}{\delta \psi_{\alpha}}=\prod_{\beta \in \widetilde{M}} \frac{\delta}{\delta \underline{a}_{\beta}} .
$$

Lemma 4.3. The Grassmann integral does not depend on the choice of the generators. More precisely, for $\underline{a}$ and $\underline{b}$ as defined in (4.1) and a transformation defined by

$$
\underline{b}=U \underline{a},
$$

where $U$ is a unitary $2|M| \times 2|M|$-matrix, we have

$$
\frac{\delta}{\delta \underline{b}}=\bar{U} \frac{\delta}{\delta \underline{a}}
$$

and, for any $\mu \in \mathcal{G}_{M}$,

$$
\prod_{\alpha \in M}\left(\frac{\delta}{\delta \bar{\psi}_{\alpha}} \frac{\delta}{\delta \psi_{\alpha}}\right) \mu(\bar{\psi}, \psi)=\prod_{\alpha \in M}\left(\frac{\delta}{\delta \bar{\chi}_{\alpha}} \frac{\delta}{\delta \chi_{\alpha}}\right) \mu(\bar{\chi}, \chi) .
$$

Proof. First we prove $\frac{\delta}{\delta \underline{b}}=\bar{U} \frac{\delta}{\delta a}$. The identity $\frac{\delta}{\delta a_{j}} a_{i}=\delta_{i j}$ follows from the properties of the generators. An equivalent identity has to be claimed for $\frac{\delta}{\delta \underline{b} \underline{b}}$. Suppose $\frac{\delta}{\delta \underline{b}}$ transforms as $\frac{\delta}{\delta \underline{b}}=V \frac{\delta}{\delta \underline{a}}$ with a $2|M| \times 2|M|$-matrix $V$. This yields

$$
\frac{\delta}{\delta b_{j}} b_{i}=\left(\sum_{\alpha \in \widetilde{M}} V_{j \alpha} \frac{\delta}{\delta a_{\alpha}}\right)\left(\sum_{\beta \in \widetilde{M}} U_{i \beta} a_{\beta}\right)=\left(U V^{T}\right)_{i j} .
$$

In other words, we have $U V^{T}=\mathbb{1}$ and, thus, $V=\bar{U}$. Finally, we can prove the invariance of the Grassmann integral. For a given set of generators 
$\left\{\bar{\psi}_{i}, \psi_{i}\right\}_{i \in M}$, any $\mu \in \mathcal{G}_{M}$ can be written as

$$
\mu \equiv \mu(\bar{\psi}, \psi)=\sum_{I, J \subseteq M} \alpha_{I J} \bar{\Psi}_{I} \Psi_{J}
$$

where $\alpha_{I J} \in \mathbb{C}$ for all $I, J \subseteq M$ and $I, J$ ordered. The Grassmann integral of $\mu$ is

$$
\int \mathrm{d}(\bar{\Psi}, \Psi) \mu(\bar{\psi}, \psi)=\int \mathrm{d}(\bar{\Psi}, \Psi) \sum_{I, J \subseteq M} a_{I J} \bar{\Psi}_{I} \Psi_{J}=\int \mathrm{d}(\bar{\Psi}, \Psi) \alpha_{M M} \bar{\Psi}_{M} \Psi_{M}
$$

since all other terms of $\mu$ do not contribute to the integral. If the decomposition of $\mu$ yields $\alpha_{M M}=0$, the Grassmann integral of $\mu$ vanishes. In this case there is nothing to show. For $\alpha_{M M} \neq 0$ we consider the transformation of $\int \mathrm{d}(\bar{\Psi}, \Psi)$ and $\bar{\Psi}_{M} \Psi_{M}$ separately. For $\int \mathrm{d}(\bar{\Psi}, \Psi)$ we use $\frac{\delta}{\delta \underline{a}_{i}} \frac{\delta}{\delta \underline{a}_{j}}=-\frac{\delta}{\delta \underline{a}_{j}} \frac{\delta}{\delta \underline{a}_{i}}$ for $i \neq j$ and express $\frac{\delta}{\delta \underline{b}}$ in terms of $\frac{\delta}{\delta \underline{a}}$ :

$$
\begin{aligned}
\left(\prod_{\alpha \in M} \frac{\delta}{\delta \bar{\chi}_{\alpha}}\right)\left(\prod_{\alpha \in M} \frac{\delta}{\delta \chi_{\alpha}}\right) & =\prod_{\beta \in \widetilde{M}} \frac{\delta}{\delta \underline{b}_{\beta}}=\sum_{\beta_{1}, \ldots, \beta_{|\widetilde{M}|} \in \widetilde{M}} \prod_{j \in \widetilde{M}} \bar{U}_{j \beta_{j}} \frac{\delta}{\delta \underline{a}_{\beta_{j}}} \\
& =\sum_{\pi \in \mathcal{S}_{\widetilde{M}}} \prod_{j \in \widetilde{M}} \bar{U}_{j \pi(j)} \frac{\delta}{\delta \underline{a}_{\pi(j)}} \\
& =\sum_{\pi \in \mathcal{S}_{\widetilde{M}}}(-1)^{\pi} \prod_{j \in \widetilde{M}} \bar{U}_{j \pi(j)} \frac{\delta}{\delta \underline{a}_{j}} \\
& =\operatorname{det}(\bar{U}) \prod_{j \in \widetilde{M}} \frac{\delta}{\delta \underline{a}_{j}}
\end{aligned}
$$

Analogously, we have

$$
\prod_{\alpha \in M} \bar{\chi}_{M} \prod_{\alpha \in M} \chi_{M}=\prod_{\beta \in \widetilde{M}} b_{\beta}=\operatorname{det}(U) \prod_{j \in \widetilde{M}} a_{j}
$$

Merging the results we obtain

$$
\left(\prod_{\alpha \in M} \frac{\delta}{\delta \bar{\chi}_{\alpha}}\right)\left(\prod_{\alpha \in M} \frac{\delta}{\delta \chi_{\alpha}}\right) \prod_{\alpha \in M} \bar{\chi}_{M} \prod_{\alpha \in M} \chi_{M}=|\operatorname{det}(U)|^{2} \prod_{j \in \widetilde{M}} \frac{\delta}{\delta \underline{a}_{j}} \prod_{j \in \widetilde{M}} a_{j}
$$

The proof is complete with $|\operatorname{det}(U)|^{2}=1$, since $U$ is unitary. 
Remark 4.4. The transformation $U$ mixes $\bar{\psi}_{i}$ 's and $\psi_{i}$ 's. For $U:=\left(\frac{u}{v} \frac{v}{u}\right)$, a transformation without mixing is given for $v=0$. In this case, $u$ has to be unitary.

For the application of the Grassmann integration on representability conditions we still need some tools, especially the definition of a product on $\mathcal{G}_{M}$ which induces the CAR on the Grassmann algebra.

Definition 4.5. For all $\mu \equiv \mu(\bar{\psi}, \psi)$ and $\eta \equiv \eta(\bar{\psi}, \psi) \in \mathcal{G}_{M}$, we define the star product $\mu \star \eta \in \mathcal{G}_{M}$ by

$$
(\mu \star \eta)(\bar{\psi}, \psi):=\int \mathrm{d}(\bar{\Phi}, \Phi) \mu(\bar{\psi}, \phi) \eta(\bar{\phi}, \psi) \mathrm{e}^{-(\bar{\Psi}, \Psi)} \mathrm{e}^{(\bar{\Psi}, \Phi)} \mathrm{e}^{-(\bar{\Phi}, \Phi)} \mathrm{e}^{(\bar{\Phi}, \Psi)} .
$$

We calculate the star product of two monomials $\mu:=\bar{\Psi}_{I} \Psi_{J}$ and $\eta:=$ $\bar{\Psi}_{K} \Psi_{L}$, which determines the star product in general, due to the linearity of the Grassmann integral.

Lemma 4.6. Let $I, J, K, L \subseteq M$. Then we have

$$
\begin{aligned}
& \left(\bar{\Psi}_{I} \Psi_{J}\right) \star\left(\bar{\Psi}_{K} \Psi_{L}\right) \\
= & \sigma_{S} \sigma_{J S} \cdot \mathrm{e}^{-(\bar{\Psi}, \Psi)} \bar{\Psi}_{I} \Psi_{J \backslash S} \bar{\Psi}_{K \backslash S} \Psi_{L} \prod_{\substack{\alpha \in M \\
\backslash(J \cup K)}}\left(1+\bar{\psi}_{\alpha} \psi_{\alpha}\right),
\end{aligned}
$$

where $S:=J \cap K$ and $\sigma_{J S}:=(-1)^{|S|\left(|J \backslash S|+\frac{|S|-1}{2}\right)}$. The sign $\sigma_{S}$ is given by the identity $\sigma_{S} \Phi_{S} \Phi_{J \backslash S} \bar{\Phi}_{S} \bar{\Phi}_{K \backslash S}=\Phi_{J} \bar{\Phi}_{K}$.

Proof. Writing $S:=J \cap K$, we face the integral

$$
\begin{aligned}
\left(\bar{\Psi}_{I} \Psi_{J}\right) \star\left(\bar{\Psi}_{K} \Psi_{L}\right)= & \sigma_{S} \cdot \mathrm{e}^{-(\bar{\Psi}, \Psi)} \bar{\Psi}_{I} \int \mathrm{d}(\bar{\Phi}, \Phi) \Phi_{S} \Phi_{J \backslash S} \bar{\Phi}_{S} \bar{\Phi}_{K \backslash S} \\
& \times \prod_{\alpha \in M}\left(1+\bar{\phi}_{\alpha} \psi_{\alpha}+\bar{\psi}_{\alpha} \phi_{\alpha}-\bar{\phi}_{\alpha} \phi_{\alpha}-\bar{\phi}_{\alpha} \phi_{\alpha} \bar{\psi}_{\alpha} \psi_{\alpha}\right) \Psi_{L},
\end{aligned}
$$

where we use

$$
\prod_{\alpha \in M}\left(1+\bar{\phi}_{\alpha} \psi_{\alpha}+\bar{\psi}_{\alpha} \phi_{\alpha}-\bar{\phi}_{\alpha} \phi_{\alpha}-\bar{\phi}_{\alpha} \phi_{\alpha} \bar{\psi}_{\alpha} \psi_{\alpha}\right)=\mathrm{e}^{(\bar{\Psi}, \Phi)} \mathrm{e}^{-(\bar{\Phi}, \Phi)} \mathrm{e}^{(\bar{\Phi}, \Psi)}
$$

as a consequence of Eq. (3.2). In the next step we write

$$
M=(M \backslash(J \cup K)) \dot{\cup}(J \backslash S) \dot{\cup}(K \backslash S) \dot{\cup} S
$$


(where $\dot{\cup}$ denotes a disjoint union) and arrive at

$$
\begin{aligned}
\left(\bar{\Psi}_{I} \Psi_{J}\right) \star\left(\bar{\Psi}_{K} \Psi_{L}\right)= & \sigma_{S} \sigma_{S J} \cdot \mathrm{e}^{-(\bar{\Psi}, \Psi)} \bar{\Psi}_{I} \int \mathrm{d}(\bar{\Phi}, \Phi) \prod_{\alpha \in S} \phi_{\alpha} \bar{\phi}_{\alpha} \\
& \times \prod_{\alpha \in J \backslash S}\left(\phi_{\alpha}+\phi_{\alpha} \bar{\phi}_{\alpha} \psi_{\alpha}\right) \prod_{\alpha \in K \backslash S}\left(\bar{\phi}_{\alpha}+\bar{\phi}_{\alpha} \bar{\psi}_{\alpha} \phi_{\alpha}\right) \\
& \times \prod_{\substack{\alpha \in M \\
\backslash(J \cup K)}}\left(1+\bar{\phi}_{\alpha} \psi_{\alpha}+\bar{\psi}_{\alpha} \phi_{\alpha}-\bar{\phi}_{\alpha} \phi_{\alpha}-\bar{\phi}_{\alpha} \phi_{\alpha} \bar{\psi}_{\alpha} \psi_{\alpha}\right) \Psi_{L} .
\end{aligned}
$$

The sign $\sigma_{J S}:=(-1)^{|S|\left(|J \backslash S|+\frac{|S|-1}{2}\right)}$ occurs due to the permutation of all $\phi$ 's in $\Phi_{S}$ with all $\phi$ 's in $\Phi_{J \backslash S}$, and $\Phi_{S} \bar{\Phi}_{S}=(-1)^{\frac{1}{2}|S|(|S|-1)}\left(\prod_{\alpha \in S} \phi_{\alpha} \bar{\phi}_{\alpha}\right)$. Now we can perform the integration and arrive at

$$
\begin{aligned}
\left(\bar{\Psi}_{I} \Psi_{J}\right) \star\left(\bar{\Psi}_{K} \Psi_{L}\right)= & \sigma_{S} \sigma_{J S} \cdot \mathrm{e}^{-(\bar{\Psi}, \Psi)} \bar{\Psi}_{I} \prod_{\alpha \in J \backslash S} \psi_{\alpha} \prod_{\alpha \in K \backslash S} \bar{\psi}_{\alpha} \\
& \times \prod_{\substack{\alpha \in M \\
\backslash(J \cup K)}}\left(1+\bar{\psi}_{\alpha} \psi_{\alpha}\right) \Psi_{L},
\end{aligned}
$$

as claimed in Eq. (4.2), since all involved sets are disjoint.

Several properties of the star product follow directly from Lemma 4.6.

Lemma 4.7. For all $\mu, \eta, \nu \in \mathcal{G}_{M}$ we have

$$
\mu \star(\eta \star \nu)=(\mu \star \eta) \star \nu .
$$

Proof. By the definition of the star product we have

$$
\begin{aligned}
& \mu \star(\eta \star \nu)= \mu(\bar{\psi}, \psi) \star \int \mathrm{d}(\bar{\Phi}, \Phi) \eta(\bar{\psi}, \phi) \nu(\bar{\phi}, \psi) \mathrm{e}^{-(\bar{\Psi}, \Psi)+(\bar{\Psi}, \Phi)-(\bar{\Phi}, \Phi)+(\bar{\Phi}, \Psi)} \\
&=\int \mathrm{d}(\bar{\Omega}, \Omega) \int \mathrm{d}(\bar{\Phi}, \Phi) \mu(\bar{\psi}, \omega) \eta(\bar{\omega}, \phi) \nu(\bar{\phi}, \psi) \\
& \times \mathrm{e}^{-(\bar{\Psi}, \Psi)+(\bar{\Psi}, \Omega)-(\bar{\Omega}, \Omega)+(\bar{\Omega}, \Phi)-(\bar{\Phi}, \Phi)+(\bar{\Phi}, \Psi)}
\end{aligned}
$$

Performing the integration with respect to $(\bar{\phi}, \phi)$ we obtain

$$
\begin{aligned}
\mu \star(\eta \star \nu)=\int & \mathrm{d}(\bar{\Omega}, \Omega) \mu(\bar{\psi}, \omega) \eta(\bar{\omega}, \psi) \mathrm{e}^{-(\bar{\Psi}, \Psi)+(\bar{\Psi}, \Omega)-(\bar{\Omega}, \Omega)+(\bar{\Omega}, \Psi)} \\
\star \nu(\bar{\psi}, \psi) &
\end{aligned}
$$


which is, in fact, $(\mu \star \eta) \star \nu$.

As for the creation and annihilation operators on $\mathcal{B}(\wedge \mathcal{H})$, there is also an implementation of the CAR for the generators of $\mathcal{G}_{M}$.

Lemma 4.8. Let $\left\{\bar{\psi}_{i}, \psi_{i}\right\}_{i \in M}$ be the generators of $\mathcal{G}_{M}$. For $\{\mu, \eta\}_{\star}:=\mu \star$ $\eta+\eta \star \mu$ we have

$$
\left\{\psi_{i}, \psi_{j}\right\}_{\star}=\left\{\bar{\psi}_{i}, \bar{\psi}_{j}\right\}_{\star}=0 \quad \text { and } \quad\left\{\bar{\psi}_{i}, \psi_{j}\right\}_{\star}=\delta_{i j} \quad \text { for any } \quad i, j \in M .
$$

Proof. The identities follow directly from Lemma 4.6 by an appropriate choice of $I, J, K$ and $L$. We observe that

$$
\mathrm{e}^{-(\bar{\Psi}, \Psi)} \prod_{\substack{\alpha \in M \\ \backslash(J \cup K)}}\left(1+\bar{\psi}_{\alpha} \psi_{\alpha}\right)=\prod_{\alpha \in J \cup K}\left(1-\bar{\psi}_{\alpha} \psi_{\alpha}\right)
$$

and conclude for the first identity with $I=K=\emptyset, J=\{i\}$, and $L=\{j\}$ in Eq. (4.2) that $S=\emptyset$ and, therefore, $\sigma_{S}=\sigma_{J S}=1$. This yields

$$
\psi_{i} \star \psi_{j}=\left(1-\bar{\psi}_{i} \psi_{i}\right) \psi_{i} \psi_{j}=\psi_{i} \psi_{j}
$$

Setting $J=\{j\}$ and $L=\{i\}$, we obtain $\psi_{j} \star \psi_{i}=\psi_{j} \psi_{i}$ and, hence, $\psi_{i} \star$ $\psi_{j}+\psi_{j} \star \psi_{i}=\psi_{i} \psi_{j}+\psi_{j} \psi_{i}=0$. Equivalently, we obtain $\bar{\psi}_{i} \bar{\psi}_{j}+\bar{\psi}_{j} \bar{\psi}_{i}=0$. For the last identity we set $J=K=\emptyset, I=\{i\}$ and $L=\{j\}$. On the one hand, Eq. (4.2) leads to

$$
\bar{\psi}_{i} \star \psi_{j}=\bar{\psi}_{i} \psi_{j}
$$

which is valid for both $i=j$ and $i \neq j$. On the other hand, with $I=L=\emptyset$, $J=\{j\}$, and $K=\{i\}$, we have to distinguish between the cases $J=K$ and $J \neq K$. For $J \neq K$ we have

$$
\psi_{j} \star \bar{\psi}_{i}=\left(1-\bar{\psi}_{i} \psi_{i}\right)\left(1-\bar{\psi}_{j} \psi_{j}\right) \psi_{j} \bar{\psi}_{i}=\psi_{j} \bar{\psi}_{i} .
$$

For $J=K$ we have $i=j$ and $S=J=K$, and thus

$$
\psi_{j} \star \bar{\psi}_{i}=\left(1-\bar{\psi}_{i} \psi_{i}\right)
$$

Together, the last two results give $\psi_{j} \star \bar{\psi}_{i}=\delta_{i j}-\bar{\psi}_{i} \psi_{j}$. Finally, we arrive at $\bar{\psi}_{i} \star \psi_{j}+\psi_{j} \star \bar{\psi}_{i}=\delta_{i j}$. We mention that in Eqs. (4.3)-(4.4) $\sigma_{S}=\sigma_{J S}=1$ due to the choice of the sets $I, J, K$ and $L$. 
By a straightforward calculation using Lemma 4.6 one can also show that for any generator $\left\{\bar{\psi}_{i}, \psi_{i}\right\}_{i \in M}$ of $\mathcal{G}_{M}$ we have the following:

Corollary 4.9. Let $\left\{\bar{\psi}_{i}, \psi_{i}\right\}_{i \in M}$ be the generators of $\mathcal{G}_{M}$. Then we have

$$
\bar{\psi}_{i_{1}} \star \cdots \star \bar{\psi}_{i_{m}} \star \psi_{j_{1}} \star \cdots \star \psi_{j_{n}}=\bar{\psi}_{i_{1}} \cdots \bar{\psi}_{i_{m}} \psi_{j_{1}} \cdots \psi_{j_{n}}
$$

Proof. We use the associativity

$$
\bar{\psi}_{i_{1}} \star \cdots \star \bar{\psi}_{i_{m}} \star \psi_{j_{1}} \star \cdots \star \psi_{j_{n}}=\left(\bar{\psi}_{i_{1}} \star \cdots \star \bar{\psi}_{i_{m}}\right) \star\left(\psi_{j_{1}} \star \cdots \star \psi_{j_{n}}\right)
$$

and calculate the brackets using Lemma 4.6. For the first bracket we set $I=\left\{i_{1}, \ldots, i_{m}\right\}$ and $J=K=L=\emptyset$ in Eq. (4.2). For the second bracket we use $I=J=K=\emptyset$ and $L=\left\{j_{1}, \ldots, j_{n}\right\}$. For both we have $\sigma_{S}=\sigma_{J S}=1$ and we conclude

$$
\bar{\psi}_{i_{1}} \star \cdots \star \bar{\psi}_{i_{m}} \star \psi_{j_{1}} \star \cdots \star \psi_{j_{n}}=\left(\bar{\psi}_{i_{1}} \cdots \bar{\psi}_{i_{m}}\right) \star\left(\psi_{j_{1}} \cdots \psi_{j_{n}}\right)
$$

The last star product can be calculated by setting $I=\left\{i_{1}, \ldots, i_{m}\right\}, L=$ $\left\{j_{1}, \ldots, j_{n}\right\}$, and $J=K=\emptyset$ in Eq. (4.2). Again, $\sigma_{S}=\sigma_{J S}=1$ and we arrive at the assertion.

We emphasize that

$$
\bar{\psi}_{i} \psi_{j}=\bar{\psi}_{i} \star \psi_{j}, \quad \text { but } \quad \psi_{i} \bar{\psi}_{j}=-\bar{\psi}_{j} \psi_{i}=-\bar{\psi}_{j} \star \psi_{i}
$$

This implies that the star product can be inserted (or skipped) only if the monomial in $\psi$ and $\bar{\psi}$ is normal-ordered (i. e., all $\bar{\psi}$ 's are to the left of all $\psi$ 's). As follows from the proof, monomials containing only $\psi$ 's or $\bar{\psi}$ 's can also be considered as normal-ordered in the sense that we can identify $\bar{\psi}_{i_{1}} \star \cdots \star \bar{\psi}_{i_{m}}$ with $\bar{\psi}_{i_{1}} \cdots \bar{\psi}_{i_{m}}$ and $\psi_{j_{1}} \star \cdots \star \psi_{j_{n}}$ with $\psi_{j_{1}} \cdots \psi_{j_{n}}$.

Lemma 4.10. Let $N \in \mathbb{N}$ and $A_{i} \in \mathcal{B}(\wedge \mathcal{H})$ for $i \in\{1, \ldots, N\}$. Then

$$
\Theta\left(A_{1} A_{2} \cdots A_{N}\right)=\Theta\left(A_{1}\right) \star \Theta\left(A_{2}\right) \star \cdots \star \Theta\left(A_{N}\right) .
$$

Proof. Due to the associativity of the star product it suffices to consider the assertion for $N=2$. We use the CAR to establish normal-order in the product $A_{1} A_{2} \in \mathcal{B}(\wedge \mathcal{H})$ and indicate this order by $\bullet A_{1} A_{2} \bullet$ For some $\underset{\substack{i_{1} \ldots i_{m} \\ j_{1} \ldots j_{n}}}{a_{i}} \in$ 
$\mathbb{C}$, we can write

$$
\bullet A_{1} A_{2}:=\sum_{m, n} \sum_{\substack{i_{1} \ldots i_{m} \in M \\ j_{1} \ldots j_{n}}} a_{i_{1} \ldots i_{m}} c_{i_{1} \ldots j_{n}}^{*} \cdots c_{i_{m}}^{*} c_{j_{1}} \cdots c_{j_{n}}
$$

and apply $\Theta$. Together with Corollary 4.9 we arrive at

$$
\Theta\left(\bullet A_{1} A_{2} \bullet\right)=\sum_{m, n} \sum_{\substack{i_{1} \ldots i_{m} \in M \\
j_{1} \ldots j_{n}}} a_{\begin{array}{c}
i_{1_{1} \ldots i_{m}} \\
j_{1} \ldots j_{n}
\end{array}} \bar{\psi}_{i_{1}} \star \cdots \star \bar{\psi}_{i_{m}} \star \psi_{j_{1}} \star \cdots \star \psi_{j_{n}} .
$$

Now we can use the $\mathrm{CAR}$ on $\mathcal{G}_{M}$ to restore the same order we had in $A_{1} A_{2}$ within the r.h.s. of Eq. (4.5) and recognize that it equals $\Theta\left(A_{1}\right) \star \Theta\left(A_{2}\right)$. In other words, we have

$$
\sum_{m, n} \sum_{\substack{i_{1} \ldots i_{m} \in M \\ j_{1} \ldots j_{n}}} a_{\substack{i_{1} \ldots i_{m} \\ j_{1} \ldots j_{n}}} \bar{\psi}_{i_{1}} \star \cdots \star \bar{\psi}_{i_{m}} \star \psi_{j_{1}} \star \cdots \star \psi_{j_{n}}=: \Theta\left(A_{1}\right) \star \Theta\left(A_{2}\right) \bullet,
$$

which gives the assertion.

We can equip $\left(\mathcal{G}_{M},+, \star\right)$ with an involution $(\cdot)^{*} \operatorname{such}$ that $\left(\mathcal{G}_{M},+, \star,^{*}\right)$ becomes a ${ }^{*}$-algebra.

Definition 4.11. For all $\mu_{i} \in \mathcal{G}_{M}, i \in \mathbb{N}$, and $c \in \mathbb{C}$, the involution $(\cdot)^{*}$ on $\left(\mathcal{G}_{M},+, \star\right)$ is defined by $\left(\psi_{i}\right)^{*}:=\bar{\psi}_{i}$ and $\left(\bar{\psi}_{i}\right)^{*}:=\psi_{i} \forall i \in M$, and

$$
\left(c \mu_{1} \cdots \mu_{n}\right)^{*}:=\bar{c} \mu_{n}^{*} \cdots \mu_{1}^{*}
$$

Remark 4.12. For $\mu \equiv \mu(\bar{\psi}, \phi):=\sum_{I, J} a_{I J} \bar{\Psi}_{I} \Phi_{J}$ and $a_{I J} \in \mathbb{C}$, the involution $\mu^{*}$ is given by

$$
\mu^{*}(\bar{\phi}, \psi)=\sum_{I, J} \bar{a}_{I J} \bar{\Phi}_{J^{\prime}} \Psi_{I^{\prime}}=\sum_{I, J}(-1)^{\frac{1}{2}|I|(|I|-1)+\frac{1}{2}|J|(|J|-1)} \bar{\alpha}_{I J} \bar{\Phi}_{J} \Psi_{I} .
$$

We emphasize that $(\mu(\bar{\psi}, \phi))^{*}=\mu^{*}(\bar{\phi}, \psi) \neq(\mu(\bar{\phi}, \psi))^{*}$.

Lemma 4.13. The involution in Definition 4.11 is compatible with $\Theta$, the Grassmann integration, and the star product:

(a) $\Theta\left((\cdot)^{*}\right)=(\Theta(\cdot))^{*}$,

(b) $\int \mathrm{d}(\bar{\Psi}, \Psi)(\cdot)^{*}=\left[\int \mathrm{d}(\bar{\Psi}, \Psi)(\cdot)\right]^{*}$, 
(c) $(\mu \star \eta)^{*}=\eta^{*} \star \mu^{*}$.

Proof. We prove (a) and (b). (c) is a consequence of (b).

(a) For any $I, J \subseteq M$, we abbreviate $C_{I}^{*}:=c_{i_{1}}^{*} \cdots c_{i_{m}}^{*}$ and $C_{J}:=c_{j_{1}} \cdots c_{j_{n}}$ and write any $A \in \mathcal{B}(\mathcal{H})$ as $A=\sum_{I, J} a_{I J} C_{I}^{*} C_{J}$ for some $a_{I J} \in \mathbb{C}$. This leads to

$$
\begin{aligned}
(\Theta(A))^{*} & =\left(\sum_{I, J} a_{I J} \bar{\Psi}_{I} \Psi_{J}\right)^{*}=\sum_{I, J} \bar{a}_{I J} \bar{\Psi}_{J^{\prime}} \Psi_{I^{\prime}}=\Theta\left(\sum_{I, J} \bar{a}_{I J} C_{J^{\prime}}^{*} C_{I^{\prime}}\right) \\
& =\Theta\left(\left(\sum_{I, J} a_{I J} C_{I}^{*} C_{J}\right)^{*}\right)=\Theta\left(A^{*}\right) .
\end{aligned}
$$

(b) We formally have $\left(\frac{\delta}{\delta \bar{\psi}_{i}} \frac{\delta}{\delta \psi_{i}}\right)^{*} \mu=\frac{\delta}{\delta \bar{\psi}_{i}} \frac{\delta}{\delta \psi_{i}} \mu$ for any fixed, but arbitrary $i \in M$ and any $\mu \in \mathcal{G}_{M}$, which gives the assertion.

(c) We calculate the 1.h.s. of (c) using (b) and Remark 4.12:

$$
\begin{aligned}
& \begin{aligned}
(\mu \star \eta)^{*} & =\int \mathrm{d}(\bar{\Phi}, \Phi) \eta^{*}(\bar{\psi}, \phi) \mu^{*}(\bar{\phi}, \psi) \mathrm{e}^{-(\bar{\Psi}, \Psi)} \mathrm{e}^{(\bar{\Psi}, \Phi)} \mathrm{e}^{-(\bar{\Phi}, \Phi)} \mathrm{e}^{(\bar{\Phi}, \Psi)} \\
& =\eta^{*} \star \mu^{*}
\end{aligned} \\
& \text { since }\left(\mathrm{e}^{(\cdot)}\right)^{*}=\mathrm{e}^{(\cdot)} .
\end{aligned}
$$

A key property of the Grassmann integral for deriving representability conditions as in the next section is the cyclicity property which has its equivalent in the cyclicity of the trace, i. e., $\operatorname{tr}(A B)=\operatorname{tr}(B A)$.

Theorem 4.14. For $\mu, \eta \in \mathcal{G}_{M}$, we have

$$
\int \mathcal{D}(\bar{\Psi}, \Psi)(\mu \star \eta)=\int \mathcal{D}(\bar{\Psi}, \Psi)(\eta \star \mu) .
$$

Proof. Without loss of generality, we can set

$$
\mu:=\bar{\Psi}_{I} \Psi_{J} \quad \text { and } \quad \eta:=\bar{\Psi}_{K} \Psi_{L}
$$


and observe with Eq. (4.2) and $T:=I \cap L$

$$
\begin{aligned}
& \int \mathcal{D}(\bar{\Psi}, \Psi) \mu \star \eta \\
= & \sigma_{S} \sigma_{T} \sigma_{J S} \int \mathcal{D}(\bar{\Psi}, \Psi) \cdot \mathrm{e}^{-(\bar{\Psi}, \Psi)} \\
& \times \bar{\Psi}_{T} \bar{\Psi}_{I \backslash T} \prod_{\alpha \in J \backslash S} \psi_{\alpha} \prod_{\alpha \in K \backslash S} \bar{\psi}_{\alpha} \prod_{\substack{\alpha \in M \\
\backslash(J \cup K)}}\left(1+\bar{\psi}_{\alpha} \psi_{\alpha}\right) \Psi_{T} \Psi_{L \backslash T} .
\end{aligned}
$$

Afterwards, we rearrange the factors and arrive at

$$
\begin{aligned}
& \int \mathcal{D}(\bar{\Psi}, \Psi) \mu \star \eta \\
= & \sigma_{S} \sigma_{T} \tilde{\sigma} \int \mathrm{d}(\bar{\Psi}, \Psi) \bar{\Psi}_{I \backslash T} \bar{\Psi}_{K \backslash S} \Psi_{J \backslash S} \Psi_{L \backslash T} \prod_{\alpha \in T} \bar{\psi}_{\alpha} \psi_{\alpha} \\
& \times \prod_{\alpha \in M}\left(1+\bar{\psi}_{\alpha} \psi_{\alpha}\right) \prod_{\substack{\alpha \in M \\
\backslash(J \cup K)}}\left(1+\bar{\psi}_{\alpha} \psi_{\alpha}\right),
\end{aligned}
$$

where $\tilde{\sigma} \in\{ \pm 1\}$ corresponds to the signs resulting from the anticommutations and is given by

$$
\tilde{\sigma}:=(-1)^{|S||J \backslash S|+|T||K \backslash S|+\frac{1}{2}|S|(|S|-1)+\frac{1}{2}|T|(|T|-1)+|T||J \backslash S|+|T||I \backslash T|+|K \backslash S||J \backslash S|} .
$$

To go on, we need some preparation. First of all, we observe that

$$
\prod_{\alpha \in M}\left(1+\bar{\psi}_{\alpha} \psi_{\alpha}\right) \prod_{\substack{\alpha \in M \\ \backslash(J \cup K)}}\left(1+\bar{\psi}_{\alpha} \psi_{\alpha}\right)=\prod_{\substack{\alpha \in M \\ \backslash(J \cup K)}}\left(1+2 \bar{\psi}_{\alpha} \psi_{\alpha}\right) \prod_{\alpha \in J \cup K}\left(1+\bar{\psi}_{\alpha} \psi_{\alpha}\right)
$$

On the one hand, we have $J \cup K=(J \backslash S) \dot{\cup}(K \backslash S) \dot{\cup} S$, which implies

$$
\prod_{\alpha \in J \cup K}\left(1+\bar{\psi}_{\alpha} \psi_{\alpha}\right) \bar{\Psi}_{K \backslash S} \Psi_{J \backslash S}=\prod_{\alpha \in S}\left(1+\bar{\psi}_{\alpha} \psi_{\alpha}\right) \bar{\Psi}_{K \backslash S} \Psi_{J \backslash S} .
$$

On the other hand, we have by the same arguments

$$
\begin{aligned}
& \prod_{\substack{\alpha \in M \\
\backslash(J \cup K)}}\left(1+2 \bar{\psi}_{\alpha} \psi_{\alpha}\right) \bar{\Psi}_{I \backslash T} \bar{\Psi}_{K \backslash S} \Psi_{J \backslash S} \Psi_{L \backslash T} \prod_{\alpha \in T} \bar{\psi}_{\alpha} \psi_{\alpha} \\
= & \prod_{\substack{\alpha \in M \\
\backslash(J \cup K \cup I \cup L)}}\left(1+2 \bar{\psi}_{\alpha} \psi_{\alpha}\right) \bar{\Psi}_{I \backslash T} \bar{\Psi}_{K \backslash S} \Psi_{J \backslash S} \Psi_{L \backslash T} \prod_{\alpha \in T} \bar{\psi}_{\alpha} \psi_{\alpha},
\end{aligned}
$$


since $I \cup L \equiv(I \backslash T) \cup(L \backslash T) \cup \dot{T}$. Consequently, our latter calculations lead in Eq. (4.6) to

$$
\begin{aligned}
& \int \mathcal{D}(\bar{\Psi}, \Psi) \mu \star \eta \\
= & \sigma_{S} \sigma_{T} \tilde{\sigma} \int \mathrm{d}(\bar{\Psi}, \Psi) \bar{\Psi}_{I \backslash T} \bar{\Psi}_{K \backslash S} \Psi_{J \backslash S} \Psi_{L \backslash T} \prod_{\alpha \in T} \bar{\psi}_{\alpha} \psi_{\alpha} \\
& \times \prod_{\alpha \in S}\left(1+\bar{\psi}_{\alpha} \psi_{\alpha}\right) \prod_{\substack{\alpha \in M \\
\backslash(J \cup K \cup I \cup L)}}\left(1+2 \bar{\psi}_{\alpha} \psi_{\alpha}\right) .
\end{aligned}
$$

Let us take a closer look at the involved sets. First of all, we observe that

(I) $K \backslash S \cap J \backslash S=\emptyset$,

(II) $I \cup(K \backslash S)=L \cup(J \backslash S)$,

(III) $I \cap(K \backslash S)=\emptyset$ and

(IV) $L \cap(J \backslash S)=\emptyset$.

In any other case we have $\int \mathcal{D}(\bar{\Psi}, \Psi) \mu \star \eta=\int \mathcal{D}(\bar{\Psi}, \Psi) \eta \star \mu=0$. These observations have some consequences:

(a) (II) and (I) $\Rightarrow(K \backslash S) \subseteq L$ and $(J \backslash S) \subseteq I \Rightarrow \exists T_{1}, T_{2} \subseteq M$, such that $I=(J \backslash S) \dot{\cup} T_{1}$ and $L=(K \backslash S) \cup \dot{\cup} T_{2}$.

(b) (III) and $I=(J \backslash S) \dot{\cup} T_{1} \Rightarrow\left((J \backslash S) \cup T_{1}\right) \cap(K \backslash S)=\emptyset \Rightarrow T_{1} \cap K \backslash S=$ $\emptyset$. Analogously: (IV) and $L=(K \backslash S) \dot{\cup} T_{1} \Rightarrow T_{2} \cap(J \backslash S)=\emptyset$.

(c) (II) and (b) $\Rightarrow T_{1}=T_{2}$, since all sets on the l.h.s. and r.h.s. of (II) are disjoint.

(d) (a), (b) and (c) $\Rightarrow L \cap I=\left((K \backslash S) \dot{\cup} T_{1}\right) \cap\left((J \backslash S) \dot{\cup} T_{2}\right)=T_{1} \cap$ $T_{2}=: T$.

Back to (a), we see that $I=(J \backslash S) \dot{\cup} T$ or $I \backslash T=J \backslash S$, and $L=(K \backslash S) \dot{\cup} T$ implies $L \backslash T=K \backslash S$. This is illustrated in the following figure.

We go on in Eq. (4.7) and take the intersection $S \cap T$ into account. The term $\prod_{\alpha \in T} \bar{\psi}_{\alpha} \psi_{\alpha} \prod_{\alpha \in S}\left(1+\bar{\psi}_{\alpha} \psi_{\alpha}\right)$ contributes to the integral as follows:

$$
\prod_{\alpha \in T \cup S} \frac{\delta}{\delta \bar{\psi}_{\alpha}} \frac{\delta}{\delta \psi_{\alpha}} \prod_{\alpha \in T} \bar{\psi}_{\alpha} \psi_{\alpha} \prod_{\beta \in S}\left(1+\bar{\psi}_{\beta} \psi_{\beta}\right)=\prod_{\alpha \in T \cup S} \frac{\delta}{\delta \bar{\psi}_{\alpha}} \frac{\delta}{\delta \psi_{\alpha}} \prod_{\alpha \in T \cup S} \bar{\psi}_{\alpha} \psi_{\alpha}
$$




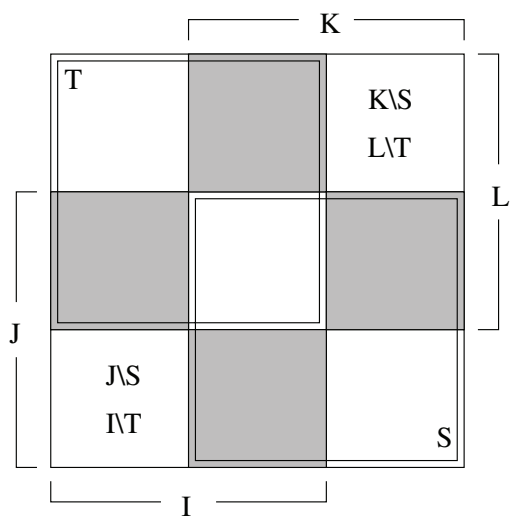

Figure 1: Chequerboard: The integrals vanish if $J \cup L \neq I \cup K . S:=J \cap K$ and $T:=I \cap L$. Grey areas represent empty subsets.

since $\prod_{\alpha \in T \cap S} \bar{\psi}_{\alpha} \psi_{\alpha} \prod_{\beta \in T \cap S}\left(1+\bar{\psi}_{\beta} \psi_{\beta}\right)=\prod_{\alpha \in T \cap S} \bar{\psi}_{\alpha} \psi_{\alpha}$ and

$$
\begin{aligned}
& \prod_{\alpha \in S \backslash(T \cap S)} \frac{\delta}{\delta \bar{\psi}_{\alpha}} \frac{\delta}{\delta \psi_{\alpha}} \prod_{\beta \in S \backslash(T \cap S)}\left(1+\bar{\psi}_{\beta} \psi_{\beta}\right) \\
= & \prod_{\alpha \in S \backslash(T \cap S)} \frac{\delta}{\delta \bar{\psi}_{\alpha}} \frac{\delta}{\delta \psi_{\alpha}} \prod_{\beta \in S \backslash(T \cap S)} \bar{\psi}_{\beta} \psi_{\beta} .
\end{aligned}
$$

This finishes our calculations and we conclude

$$
\begin{aligned}
\int \mathcal{D}(\bar{\Psi}, \Psi) \mu \star \eta= & \sigma_{S} \sigma_{T} \tilde{\sigma} \int \mathrm{d}(\bar{\Psi}, \Psi) \prod_{\alpha \in T \cup S} \bar{\psi}_{\alpha} \psi_{\alpha} \\
& \times \prod_{\substack{\alpha \in M \\
\backslash(J \cup K \cup I \cup L)}}\left(1+2 \bar{\psi}_{\alpha} \psi_{\alpha}\right) \bar{\Psi}_{I \backslash T} \bar{\Psi}_{K \backslash S} \Psi_{J \backslash S} \Psi_{L \backslash T}
\end{aligned}
$$

The r.h. s. of the assertion in Theorem 4.14 can be calculated analogously. The result is

$$
\begin{aligned}
\int \mathcal{D}(\bar{\Psi}, \Psi) \eta \star \mu= & \sigma_{T} \sigma_{S} \widehat{\sigma} \int \mathrm{d}(\bar{\Psi}, \Psi) \prod_{\alpha \in S \cup T} \bar{\psi}_{\alpha} \psi_{\alpha} \\
& \times \prod_{\substack{\alpha \in M \\
\backslash(J \cup K \cup I \cup L)}}\left(1+2 \bar{\psi}_{\alpha} \psi_{\alpha}\right) \bar{\Psi}_{K \backslash S} \bar{\Psi}_{I \backslash T} \Psi_{L \backslash T} \Psi_{J \backslash S},
\end{aligned}
$$


where the sign resulting from the anticommutations is

$$
\widehat{\sigma}:=(-1)^{|T||L \backslash T|+|S||L \backslash T|+\frac{1}{2}|S|(|S|-1)+\frac{1}{2}|T|(|T|-1)+|S||I \backslash T|+|S||K \backslash S|+|I \backslash T||L \backslash T|} .
$$

The 1. h. s. and the r. h. s. of the asserted identy in Theorem 4.14 are symmetric with respect to the involved sets. The proof is complete by the observation

$$
\tilde{\sigma}=\widehat{\sigma}=(-1)^{\frac{1}{2}|S|(|S|-1)+\frac{1}{2}|T|(|T|-1)+|K \backslash S||J \backslash S|+|T||K \backslash S|+|S||J \backslash S|},
$$

which follows from $I \backslash T=J \backslash S$ and $L \backslash T=K \backslash S$.

Remark 4.15. The integral on the r.h.s. of Eq. (4.8) can be calculated explicitly. Abbreviating $s_{Q}:=\frac{1}{2}|Q|(|Q|-1)$ for $Q \subseteq M$, we have

$$
\begin{aligned}
\int \mathcal{D}(\bar{\Psi}, \Psi) \mu \star \eta= & \sigma_{S} \sigma_{T}(-1)^{s_{S}+s_{T}+|T||K \backslash S|+|S||J \backslash S|+s_{I \backslash T}+s_{K \backslash S}} \\
& \times \int \mathrm{d}(\bar{\Psi}, \Psi) \prod_{\alpha \in I \backslash T} \bar{\psi}_{\alpha} \psi_{\alpha} \prod_{\alpha \in K \backslash S} \bar{\psi}_{\alpha} \psi_{\alpha} \\
& \times \prod_{\alpha \in T \cup S} \bar{\psi}_{\alpha} \psi_{\alpha} \prod_{\alpha \in M \backslash(I \cup K)}\left(1+2 \bar{\psi}_{\alpha} \psi_{\alpha}\right) \\
= & \sigma_{S} \sigma_{T}(-1)^{s_{S}+s_{T}+|T||K \backslash S|+|S||J \backslash S|+s_{I \backslash T}+s_{K \backslash S}} \\
& \times(-1)^{|I \backslash T|+|K \backslash S|+|T \cup S|}(-2)^{|M|-|I \cup K|} .
\end{aligned}
$$

With $|I \backslash T|+|K \backslash S|+|T \cup S|=|I \cup K|$ we obtain

$$
\int \mathcal{D}(\bar{\Psi}, \Psi) \mu \star \eta=\sigma_{S} \sigma_{T}(-1)^{s_{J}+s_{L}} 2^{|M|-|I \cup K|}
$$

for $\mu:=\bar{\Psi}_{I} \Psi_{J}$ and $\eta:=\bar{\Psi}_{K} \Psi_{L}$.

Remark 4.16. A consequence of Lemma 4.7 and Theorem 4.14 is the invariance of the Grassmann integral with respect to cyclic permutations of the integrand:

$$
\int \mathrm{d}(\bar{\Psi}, \Psi)\left(\mu_{1} \star \mu_{2} \star \cdots \star \mu_{N}\right)=\int \mathrm{d}(\bar{\Psi}, \Psi)\left(\mu_{2} \star \cdots \star \mu_{N} \star \mu_{1}\right) .
$$

This also holds true for $\int \mathcal{D}(\bar{\Psi}, \Psi)(\cdot)$, since $\mathrm{e}^{2(\bar{\Psi}, \Psi)}$ commutes with any $\mu \in \mathcal{G}_{M}$.

Given an involution on $\left(\mathcal{G}_{M},+, \star\right)$, we define the property of positivity on $\mathcal{G}_{M}$ as follows: 
Definition 4.17. We call $\mu \in \mathcal{G}_{M}$ positive semi-definite, shortly $\mu \geq 0$, if there exists an $\eta \in \mathcal{G}_{M}$ such that

$$
\mu=\eta^{*} \star \eta
$$

Approaching the problem of representability by Grassmann integration, an important result is the following theorem.

Theorem 4.18. For any $\mu \in \mathcal{G}_{M}$ with $\mu \geq 0$ we have

$$
(-1)^{|M|} \int \mathcal{D}(\bar{\Psi}, \Psi) \mu \geq 0
$$

Proof. We use an induction in $|M| \in \mathbb{N}$. For this purpose, we write any $\xi \in \mathcal{G}_{M+1}:=\operatorname{span}\left\{\bar{\psi}_{1}, \ldots, \bar{\psi}_{|M|}, \bar{\psi}_{|M|+1}, \psi_{1}, \ldots, \psi_{|M|}, \psi_{|M|+1}\right\}$ as

$$
\xi=\eta_{00}+\eta_{01} \psi_{|M|+1}+\bar{\psi}_{|M|+1} \eta_{10}+\bar{\psi}_{|M|+1} \eta_{11} \psi_{|M|+1}
$$

for normal-ordered $\eta_{00}, \eta_{01}, \eta_{10}, \eta_{11} \in \mathcal{G}_{M}$. We indicate integration with respect to a certain index set $M$ by writing $\int \mathrm{d}_{M}(\bar{\Psi}, \Psi)$ and $\int \mathcal{D}_{M}(\bar{\Psi}, \Psi)$, respectively. Furthermore, we recall that

$$
\begin{aligned}
\mathrm{e}^{E_{M}} & :=\mathrm{e}^{(\bar{\Psi}, \Psi)} \mathrm{e}^{(\bar{\Psi}, \Phi)} \mathrm{e}^{-(\bar{\Phi}, \Phi)} \mathrm{e}^{(\bar{\Phi}, \Psi)} \\
& =\prod_{\alpha \in M}\left(1-\bar{\phi}_{\alpha} \phi_{\alpha}+\bar{\psi}_{\alpha} \psi_{\alpha}+\bar{\phi}_{\alpha} \psi_{\alpha}+\bar{\psi}_{\alpha} \phi_{\alpha}-2 \bar{\psi}_{\alpha} \psi_{\alpha} \bar{\phi}_{\alpha} \phi_{\alpha}\right) .
\end{aligned}
$$

In order to show Eq. (4.10) for $|M|=0$, we consider $\mu:=a^{*} \star a \in \mathcal{G}_{0}$ with $a \in \mathbb{C}$, and observe that with $\int \mathcal{D}_{0}(\bar{\Psi}, \Psi)=1$ the 1.h.s. of Eq. (4.10) is nonnegative,

$$
\int \mathcal{D}_{0}(\bar{\Psi}, \Psi) \mu=|a|^{2} \geq 0 .
$$

Now we assume that Eq. (4.10) holds for $|M|$ and consider the l. h. s. of (4.10) for $|M|+1$ and $\mu=\xi^{*} \star \xi$. We abbreviate $\psi_{|M|+1} \equiv \psi^{\prime}$ and $\bar{\psi}_{|M|+1} \equiv{\overline{\psi^{\prime}}}_{\text {. }}$

$$
\begin{aligned}
& (-1)^{|M|+1} \int \mathcal{D}_{M+1}(\bar{\Psi}, \Psi)\left(\xi^{*} \star \xi\right) \\
= & (-1)^{|M|+1} \int \mathcal{D}_{M+1}(\bar{\Psi}, \Psi) \times\left[\eta_{00}^{*} \star \eta_{00}+\eta_{00}^{*} \star\left(\overline{\psi^{\prime}} \eta_{11} \psi^{\prime}\right)\right. \\
& +\left(\overline{\psi^{\prime}} \eta_{01}^{*}\right) \star\left(\eta_{01} \psi^{\prime}\right)+\left(\eta_{10}^{*} \psi^{\prime}\right) \star\left(\overline{\psi^{\prime}} \eta_{10}\right) \\
& \left.+\left(\overline{\psi^{\prime}} \eta_{11}^{*} \psi^{\prime}\right) \star \eta_{00}+\left(\overline{\psi^{\prime}} \eta_{11}^{*} \psi^{\prime}\right) \star\left(\overline{\psi^{\prime}} \eta_{11} \psi^{\prime}\right)\right] .
\end{aligned}
$$


Other terms like $\int \mathcal{D}_{M+1}(\bar{\Psi}, \Psi) \eta_{00}^{*} \star\left(\eta_{01} \psi^{\prime}\right)$ vanish. This can be seen by Eq. (4.7), since, in this case, $I \cup K \neq J \cup L$.

In the next step, we use the definition of the star product and the identity $\int \mathrm{d}_{M+1}(\bar{\Psi}, \Psi)=\int \mathrm{d}_{M}(\bar{\Psi}, \Psi) \frac{\delta}{\delta \overline{\psi^{\prime}}} \frac{\delta}{\delta \psi^{\prime}}$ to carry out all integrations with respect to $\psi^{\prime}$ and $\overline{\psi^{\prime}}$. We exemplify this step by the last term on the r. h.s. of Eq. (4.11):

$$
\begin{aligned}
& (-1)^{|M|+1} \int \mathcal{D}_{M+1}(\bar{\Psi}, \Psi)\left(\overline{\psi^{\prime}} \eta_{11}^{*} \psi^{\prime}\right) \star\left(\overline{\psi^{\prime}} \eta_{11} \psi^{\prime}\right) \\
= & (-1)^{|M|+1} \int \mathrm{d}_{M+1}(\bar{\Psi}, \Psi) \int \mathrm{d}_{M+1}(\bar{\Phi}, \Phi) \\
& \times \overline{\psi^{\prime}} \eta_{11}^{*}(\bar{\psi}, \phi) \phi^{\prime} \overline{\phi^{\prime}} \eta_{11}(\bar{\phi}, \psi) \psi^{\prime} \mathrm{e}^{E_{M+1}} .
\end{aligned}
$$

Since $\eta_{11}^{*}(\bar{\psi}, \phi) \eta_{11}(\bar{\phi}, \psi)$ is even in the $(\bar{\psi}, \psi, \bar{\phi}, \phi)$-variables, we continue with

$$
\begin{aligned}
& (-1)^{|M|+1} \int \mathcal{D}_{M+1}(\bar{\Psi}, \Psi)\left(\overline{\psi^{\prime}} \eta_{11}^{*} \psi^{\prime}\right) \star\left(\overline{\psi^{\prime}} \eta_{11} \psi^{\prime}\right) \\
= & (-1)^{|M|+1} \int \mathrm{d}_{M}(\bar{\Psi}, \Psi) \int \mathrm{d}_{M}(\bar{\Phi}, \Phi) \eta_{11}^{*}(\bar{\psi}, \phi) \eta_{11}(\bar{\phi}, \psi) \mathrm{e}^{E_{M}} \\
& \times \frac{\delta}{\delta \overline{\phi^{\prime}}} \frac{\delta}{\delta \phi^{\prime}} \frac{\delta}{\delta \overline{\psi^{\prime}}} \frac{\delta}{\delta \psi^{\prime}} \overline{\psi^{\prime}} \phi^{\prime} \overline{\phi^{\prime}} \psi^{\prime}\left(1-\overline{\phi^{\prime}} \phi^{\prime}+\overline{\psi^{\prime}} \psi^{\prime}+\overline{\phi^{\prime}} \psi^{\prime}+\overline{\psi^{\prime}} \phi^{\prime}-2 \overline{\psi^{\prime}} \psi^{\prime} \overline{\phi^{\prime}} \phi^{\prime}\right) \\
= & (-1)^{|M|+2} \int \mathcal{D}_{M}(\bar{\Psi}, \Psi) \eta_{11}^{*} \star \eta_{11} .
\end{aligned}
$$

By analogous calculations, we obtain

$$
\begin{aligned}
(-1)^{|M|+1} \int \mathcal{D}_{M+1}(\bar{\Psi}, \Psi)\left(\xi^{*} \star \xi\right) & \\
=(-1)^{|M|+2} \int \mathcal{D}_{M}(\bar{\Psi}, \Psi)[ & 2 \eta_{00}^{*} \star \eta_{00}+\eta_{00}^{*} \star \widetilde{\eta}_{11}+\eta_{01}^{*} \star \eta_{01} \\
& \left.+\eta_{10}^{*} \star \eta_{10}+\widetilde{\eta}_{11}^{*} \star \eta_{00}+\eta_{11}^{*} \star \eta_{11}\right],
\end{aligned}
$$

where $\widetilde{\eta}_{11}:=\sum_{I, J}(-1)^{|I|+|J|} a_{I J} \bar{\Psi}_{I} \Psi_{J} \in \mathcal{G}_{M}$ if $\eta_{11}:=\sum_{I, J} a_{I J} \bar{\Psi}_{I} \Psi_{J}$ for some $a_{I J} \in \mathbb{C}$. $\widetilde{\eta}_{11}$ occurs due to the anticommutations of $\psi_{M+1}$ with $\eta_{11}^{*}$ and of $\bar{\psi}_{M+1}$ with $\eta_{11}$ in the second and the fifth term on the r.h.s. of Eq. (4.11), 
respectively. Observing that

$$
\begin{aligned}
& \int \mathcal{D}_{M}(\bar{\Psi}, \Psi) \widetilde{\eta}_{11}^{*} \star \widetilde{\eta}_{11} \\
= & \sum_{I, J, K, L} a_{I J} \bar{a}_{L K}(-1)^{|I|+|J|+|K|+|L|} \int \mathcal{D}_{M}(\bar{\Psi}, \Psi)\left(\bar{\Psi}_{I} \Psi_{J}\right) \star\left(\bar{\Psi}_{K} \Psi_{L}\right) \\
= & \int \mathcal{D}_{M}(\bar{\Psi}, \Psi) \eta_{11}^{*} \star \eta_{11}
\end{aligned}
$$

since $|I|+|J|+|K|+|L|$ is even (otherwise both integrals vanish), we finally conclude

$$
\begin{aligned}
& (-1)^{|M|+1} \int \mathcal{D}_{M+1}(\bar{\Psi}, \Psi)\left(\xi^{*} \star \xi\right) \\
= & (-1)^{|M|+2} \int \mathcal{D}_{M}(\bar{\Psi}, \Psi) \\
& \times\left[\eta_{00}^{*} \star \eta_{00}+\left(\eta_{00}+\widetilde{\eta}_{11}\right)^{*} \star\left(\eta_{00}+\widetilde{\eta}_{11}\right)+\eta_{01}^{*} \star \eta_{01}+\eta_{10}^{*} \star \eta_{10}\right],
\end{aligned}
$$

which is nonnegative by the induction hypothesis.

Finally, we can express the trace of an operator of $\mathcal{B}(\wedge \mathcal{H})$ and, due to Lemma 4.10, the trace of a product of such operators as a Grassmann integral.

Theorem 4.19. For all $A \in \mathcal{B}(\wedge \mathcal{H})$ we have

$$
\operatorname{tr}_{\wedge \mathcal{H}}(A)=(-1)^{|M|} \int \mathcal{D}(\bar{\Psi}, \Psi) \Theta(A) .
$$

Proof. Without loss of generality we may assume that $A \in \mathcal{B}(\wedge \mathcal{H})$ is normalordered. Since the trace and the Grassmann integral are linear, it suffices to consider $\operatorname{tr}_{\wedge \mathcal{H}}\left(c_{i_{1}}^{*} \cdots c_{i_{m}}^{*} c_{j_{1}} \cdots c_{j_{n}}\right)$, where $I:=\left\{i_{1}, \ldots, i_{m}\right\}$ and $J:=$ $\left\{j_{1}, \ldots, j_{n}\right\}$ are ordered. For $I \neq J$ both the l.h.s. and the r. h. s. of Eq. (4.12) vanish. For $I=J$, the l.h. s. of Eq. (4.12) is given by

$$
\operatorname{tr}_{\wedge \mathcal{H}}\left(c_{i_{1}}^{*} \cdots c_{i_{m}}^{*} c_{i_{1}} \cdots c_{i_{m}}\right)=(-1)^{\frac{1}{2}|I|(|I|-1)} 2^{|M|-|I|} .
$$


We have $\Theta\left(c_{i_{1}}^{*} \cdots c_{i_{m}}^{*} c_{i_{1}} \cdots c_{i_{m}}\right)=\bar{\psi}_{i_{1}} \cdots \bar{\psi}_{i_{m}} \psi_{i_{1}} \cdots \psi_{i_{m}}$ on the r.h.s. of the asserted Eq. (4.12) and, thus,

$$
\begin{aligned}
\int \mathcal{D}(\bar{\Psi}, \Psi) \bar{\psi}_{i_{1}} \cdots \bar{\psi}_{i_{m}} \psi_{i_{1}} \cdots \psi_{i_{m}} & =(-1)^{\frac{1}{2}|I|(|I|+1)} \int \mathcal{D}(\bar{\Psi}, \Psi) \prod_{\alpha=1}^{m}\left(\psi_{i_{\alpha}} \bar{\psi}_{i_{\alpha}}\right) \\
& =(-1)^{|M|}(-1)^{\frac{1}{2}|I|(|I|+1)} 2^{|M|-|I|}
\end{aligned}
$$

since $\prod_{\alpha \in I}\left(\psi_{\alpha} \bar{\psi}_{\alpha}\right) \mathrm{e}^{2(\bar{\Psi}, \Psi)}=\prod_{\alpha \in I}\left(\psi_{\alpha} \bar{\psi}_{\alpha}\right) \prod_{\alpha \in M \backslash I}\left(1+2 \bar{\psi}_{\alpha} \psi_{\alpha}\right)$ and, therefore,

$$
\prod_{\alpha \in M}\left(\frac{\delta}{\delta \bar{\psi}} \frac{\delta}{\delta \psi}\right) \prod_{\alpha \in I}\left(\psi_{\alpha} \bar{\psi}_{\alpha}\right) \mathrm{e}^{2(\bar{\Psi}, \Psi)}=(-2)^{|M|-|I|}
$$

The proof is complete by $(-2)^{|M|-|I|}=(-1)^{|M|}(-1)^{|I|} 2^{|M|-|I|}$.

Due to the restriction to a Hilbert space with even dimension, we henceforth skip the factor $(-1)^{|M|}$.

\section{Representability conditions from Grassmann integrals}

The last section allows for an application of the Grassmann integration on the problem of representability for fermion systems. In particular, we are interested in necessary conditions for the 1- and 2-pdm to have their origin in a density matrix $\rho[2]$. In the language of Grassmann integration we call the equivalents of density matrices Grassmann densities.

Definition 5.1. A Grassmann variable $\vartheta^{*} \star \vartheta \in \mathcal{G}_{M}$ is called Grassmann density if it is normalized, i. e., fulfills

$$
\int \mathcal{D}(\bar{\Psi}, \Psi) \vartheta^{*} \star \vartheta=1
$$

By definition, the Grassmann density is positive semi-definite and selfadjoint. For a given state $\rho$, the map $\Theta$ immediately provides $\vartheta^{*} \star \vartheta$, namely $\vartheta^{*} \star \vartheta=\Theta(\rho)$. Thanks to the product rule for $\Theta$ and the positive semidefiniteness of $\rho$, we also have $\vartheta^{*} \star \vartheta=\Theta\left(\rho^{\frac{1}{2}} \rho^{\frac{1}{2}}\right)=\Theta\left(\rho^{\frac{1}{2}}\right) \star \Theta\left(\rho^{\frac{1}{2}}\right)$. $\Theta$ is a bijection and compatible with the involution. This implies that $\vartheta=\Theta\left(\rho^{\frac{1}{2}}\right)$. Given a Grassmann density, we can formulate the problem of representability by Grassmann integrals using the trace formula (4.12). 
Definition 5.2. Let $\left\{\bar{\psi}_{i}, \psi_{i}\right\}_{i \in M}$ be the generators of $\mathcal{G}_{M}$ and associate $\left\{\psi_{i}\right\}_{i \in M}$ with a fixed ONB of $\mathcal{H}$. The 1-pdm $\gamma_{\vartheta} \in \mathcal{B}(\mathcal{H})$ and the 2-pdm $\Gamma_{\vartheta} \in \mathcal{B}(\mathcal{H} \otimes \mathcal{H})$ of a Grassmann density $\vartheta^{*} \star \vartheta$ are defined by their matrix elements

$$
\begin{aligned}
\left\langle\psi_{k}, \gamma_{\vartheta} \psi_{l}\right\rangle & :=\int \mathcal{D}(\bar{\Psi}, \Psi) \vartheta^{*} \star \vartheta \star \bar{\psi}_{l} \star \psi_{k} \text { and } \\
\left\langle\psi_{m} \otimes \psi_{n}, \Gamma_{\vartheta}\left(\psi_{l} \otimes \psi_{k}\right)\right\rangle & :=\int \mathcal{D}(\bar{\Psi}, \Psi) \vartheta^{*} \star \vartheta \star \bar{\psi}_{k} \star \bar{\psi}_{l} \star \psi_{m} \star \psi_{n} .
\end{aligned}
$$

Applying the trace formula (4.12) on Eqs. (5.1) and (5.2), respectively, we observe that

$$
\begin{aligned}
\left\langle\psi_{k}, \gamma_{\rho} \psi_{l}\right\rangle & =\operatorname{tr}_{\wedge \mathcal{H}}\left(\Theta^{-1}\left(\vartheta^{*} \star \vartheta\right) c_{l}^{*} c_{k}\right) \quad \text { and } \\
\left\langle\psi_{m} \otimes \psi_{n}, \Gamma_{\rho}\left(\psi_{l} \otimes \psi_{k}\right)\right\rangle & =\operatorname{tr}_{\wedge \mathcal{H}}\left(\Theta^{-1}\left(\vartheta^{*} \star \vartheta\right) c_{l}^{*} c_{k}^{*} c_{n} c_{m}\right),
\end{aligned}
$$

which agrees with the common definition of the 1- and 2-pdm [2] if we interpret $\Theta^{-1}\left(\vartheta^{*} \star \vartheta\right)=\left(\Theta^{-1}(\vartheta)\right)^{*} \Theta^{-1}(\vartheta)$ as a density matrix $\rho \in \mathcal{B}(\wedge \mathcal{H})$. Then the problem of representability can be formulated as follows:

Definition 5.3. We call $(\gamma, \Gamma) \in \mathcal{B}(\mathcal{H}) \times \mathcal{B}(\mathcal{H} \otimes \mathcal{H})$ representable if there exists a Grassmann density $\vartheta^{*} \star \vartheta$ such that $(\gamma, \Gamma)=\left(\gamma_{\vartheta}, \Gamma_{\vartheta}\right)$.

\subsection{Conditions on the one-particle density matrix}

The lower and upper bound for the eigenvalues of the 1-pdm $\gamma_{\vartheta}$ of a Grassmann state $\vartheta^{*} \star \vartheta$ arise directly from the definition of the 1-pdm (see [2] for further details). Here, we would like to derive the conditions by Grassmann integration. To this end, we consider certain subspaces of $\mathcal{G}_{M}$.

Definition 5.4. For any $n \in \mathbb{N}, n \leq|M|$, we define the subspace

$$
\mathcal{G}_{M}^{(n)}:=\operatorname{span}\left\{\bar{\Psi}_{I} \Psi_{J}|I, J \subseteq M,| I|,| J \mid \leq n\right\} \subseteq \mathcal{G}_{M}
$$

Bounds for the 1-pdm rise by considering $\mathcal{G}_{M}^{(1)}$. In what follows, we call conditions derived by considering $\mathcal{G}_{M}^{(n)}$ "conditions of $n$-th order".

Lemma 5.5. Theorem 4.18 implies

$$
\gamma_{\vartheta} \geq 0
$$


Proof. Let $\left\{\bar{\psi}_{i}, \psi_{i}\right\}_{i \in M}$ be the generators of $\mathcal{G}_{M}$ and $\alpha_{k} \in \mathbb{C} \forall k \in M$. In Theorem 4.18, we use Eq. (4.9) with $\eta:=\phi \star \vartheta^{*}$ and $\phi:=\sum_{k \in M} \alpha_{k} \psi_{k} \in \mathcal{G}_{M}$. We observe that $\phi^{*}=\sum_{k \in M} \bar{\alpha}_{k} \bar{\psi}_{k}$ and $\eta^{*}=\left(\phi \star \vartheta^{*}\right)^{*}=\vartheta \star \phi^{*}$ with the involution $(\cdot)^{*}$ on $\mathcal{G}_{M}$. This leads to

$$
0 \leq \int \mathcal{D}(\bar{\Psi}, \Psi) \eta^{*} \star \eta=\sum_{k, l \in M} \bar{\alpha}_{k} \alpha_{l} \int \mathcal{D}(\bar{\Psi}, \Psi) \vartheta^{*} \star \vartheta \star \bar{\psi}_{k} \star \psi_{l}=\left\langle f, \gamma_{\vartheta} f\right\rangle
$$

where $f:=\sum_{i \in M} \bar{\alpha}_{i} \psi_{i} \in \mathcal{H}$ is arbitrary.

The upper bound for $\gamma_{\vartheta}$ is given by another choice of $\eta$.

Lemma 5.6. Theorem 4.18 implies

$$
\gamma_{\vartheta} \leq \mathbb{1}
$$

Proof. The bound can be proved by following the steps of the proof of the lower bound. Again, we have $\alpha_{k} \in \mathbb{C} \forall k \in M$ and set $\phi^{*}=\sum_{k \in M} \bar{\alpha}_{k} \bar{\psi}_{k} \in \mathcal{G}_{M}$ and, this time, $\eta^{*}=\left(\phi^{*} \star \vartheta\right)^{*}=\vartheta^{*} \star \phi$. Before we go on, we observe that

$$
\phi \star \phi^{*}=\sum_{k, l \in M} \alpha_{k} \bar{\alpha}_{l} \psi_{k} \star \bar{\psi}_{l}=\sum_{k \in M} \bar{\alpha}_{k} \alpha_{k}-\sum_{k, l \in M} \alpha_{k} \bar{\alpha}_{l} \bar{\psi}_{l} \star \psi_{k}
$$

by the CAR on $\mathcal{G}_{M}$ given in Lemma 4.8. Inserting this into the inequality of Theorem 4.18 and using the associativity of the star product, we obtain

$$
\begin{aligned}
0 & \leq \int \mathcal{D}(\bar{\Psi}, \Psi) \eta^{*} \star \eta \\
& =\sum_{k \in M}\left|\alpha_{k}\right|^{2}-\sum_{k, l \in M} \bar{\alpha}_{l} \alpha_{k} \int \mathcal{D}(\bar{\Psi}, \Psi) \vartheta^{*} \star \vartheta \star \bar{\psi}_{l} \star \psi_{k} \\
& =\left\langle g,\left(\mathbb{1}-\gamma_{\vartheta}\right) g\right\rangle
\end{aligned}
$$

where we have used $\int \mathcal{D}(\bar{\Psi}, \Psi) \vartheta^{*} \star \vartheta=1$ and $g:=\sum_{k \in M} \bar{\alpha}_{k} \psi_{k} \in \mathcal{H}$.

Considering the subspace $\mathcal{G}_{M}^{(1)}$, we can summarize our last two results.

Theorem 5.7. Let $\vartheta \star \vartheta^{*}$ be a Grassmann density and $\gamma_{\vartheta}$ its 1-pdm. Then the following statements are equivalent:

(a) $0 \leq \gamma_{\vartheta} \leq \mathbb{1}$ 
(b) $\forall \mu \in \mathcal{G}_{M}^{(1)}: \int \mathcal{D}(\bar{\Psi}, \Psi) \vartheta^{*} \star \vartheta \star \mu \geq 0$.

Proof. In Theorem 3.1 of [2], the analogue of this theorem has been shown for polynomials in creation and annihilation operators of degree lower than or equal to two. With the bijection $\Theta$, we have a one-to-one mapping between the space of polynomials of degree lower than or equal to two and $\mathcal{G}_{M}^{(1)}$.

\subsection{G-, P-, and Q-Condition}

We proceed with representability conditions of second order by considering $\mathcal{G}_{M}^{(2)}$ and a star-product of $\bar{\psi}$ and $\psi$, in this case, for example $\phi:=$ $\sum_{k, l \in M} \alpha_{k l} \psi_{k} \star \psi_{l} \in \mathcal{G}_{M}$ with $\alpha_{k l} \in \mathbb{C} \forall k, l \in M$. This time, we are interested in conditions on $\Gamma_{\vartheta}$ and use the Grassmann integration to rewrite the matrix elements of the 2-pdm as in Eq. (5.2). The first condition is the P-Condition.

Lemma 5.8. Theorem 4.18 implies the P-Condition

$$
\Gamma_{\vartheta} \geq 0
$$

Proof. The proof is similar to the one in the last subsection. Setting $\eta:=$ $\phi \star \vartheta^{*}, \eta^{*}=\left(\phi \star \vartheta^{*}\right)^{*}=\vartheta \star \phi^{*}$, and $\phi:=\sum_{k, l \in M} \alpha_{k l} \psi_{k} \star \psi_{l} \in \mathcal{G}_{M}$ with $\alpha_{k l} \in$ $\mathbb{C} \forall k, l \in M$, we arrive at

$$
\begin{aligned}
0 & \leq \int \mathcal{D}(\bar{\Psi}, \Psi) \eta^{*} \star \eta \\
& =\sum_{k, l, m, n \in M} \bar{\alpha}_{k l} \alpha_{m n} \int \mathcal{D}(\bar{\Psi}, \Psi) \vartheta^{*} \star \vartheta \star \bar{\psi}_{l} \star \bar{\psi}_{k} \star \psi_{m} \star \psi_{n} \\
& =\left\langle F, \Gamma_{\vartheta} F\right\rangle
\end{aligned}
$$

where $F:=\sum_{k, l \in M} \bar{\alpha}_{k l}\left(\psi_{m} \otimes \psi_{n}\right) \in \mathcal{H} \otimes \mathcal{H}$ is arbitrary.

The Q-Condition is the next representability condition we want to deduce. In order to obtain a convenient formulation of this condition, we use an exchange operator on $\mathcal{B}(\mathcal{H} \otimes \mathcal{H})$ which is defined by $\operatorname{Ex}(f \otimes g):=g \otimes f$ for $f, g \in \mathcal{H}$.

Lemma 5.9. Theorem 4.18 implies the Q-Condition

$$
\Gamma_{\vartheta}+(\mathbb{1}-\operatorname{Ex})\left(\mathbb{1} \otimes \mathbb{1}-\gamma_{\vartheta} \otimes \mathbb{1}-\mathbb{1} \otimes \gamma_{\vartheta}\right) \geq 0
$$


Proof. With $\phi:=\sum_{k, l \in M} \bar{\alpha}_{k l} \bar{\psi}_{k} \star \bar{\psi}_{l} \in \mathcal{G}_{M}, \alpha_{k l} \in \mathbb{C} \forall k, l \in M$, and $\eta=\phi \star \vartheta^{*}$, we have

$$
\begin{aligned}
0 & \leq \int \mathcal{D}(\bar{\Psi}, \Psi) \eta^{*} \star \eta \\
& =\sum_{k, l, m, n \in M} \bar{\alpha}_{k l} \alpha_{m n} \int \mathcal{D}(\bar{\Psi}, \Psi) \vartheta^{*} \star \vartheta \star \psi_{n} \star \psi_{m} \star \bar{\psi}_{k} \star \bar{\psi}_{l} .
\end{aligned}
$$

Aiming for an expression in terms of $\Gamma$ and $\gamma$, we establish normal-ordering using the CAR:

$$
\begin{aligned}
\psi_{n} \star \psi_{m} \star \bar{\psi}_{k} \star \bar{\psi}_{l}= & \delta_{m k} \delta_{n l}-\delta_{n k} \delta_{m l}+\delta_{n k} \bar{\psi}_{l} \star \psi_{m} \\
& -\delta_{m k} \bar{\psi}_{l} \star \psi_{n}+\delta_{n l} \bar{\psi}_{k} \star \psi_{l} \\
& -\delta_{m l} \bar{\psi}_{k} \star \psi_{n}-\bar{\psi}_{k} \star \bar{\psi}_{l} \star \psi_{n} \star \psi_{m}
\end{aligned}
$$

As in the proof of Lemma 5.8, we write an arbitrary $G \in \mathcal{H} \otimes \mathcal{H}$ as $G:=$ $\sum_{k, l \in M} \alpha_{k l}\left(\psi_{k} \otimes \psi_{l}\right)$ for some $\alpha_{k l} \in \mathbb{C}$. Thus, we have $\sum_{k, l, m, n \in M} \bar{\alpha}_{k l} \alpha_{m n} \delta_{k m} \delta_{l n}=$ $\langle G, \mathbb{1} G\rangle$ and $\sum_{k, l, m, n \in M} \bar{\alpha}_{k l} \alpha_{m n} \delta_{k n} \delta_{l m}=\langle G$, Ex $G\rangle$. With Eqs. (5.1) and (5.2) we find

$$
0 \leq\left\langle G,\left(\Gamma_{\vartheta}+(\mathbb{1}-\mathrm{Ex})\left(\mathbb{1} \otimes \mathbb{1}-\gamma_{\vartheta} \otimes \mathbb{1}-\mathbb{1} \otimes \gamma_{\vartheta}\right)\right) G\right\rangle
$$

by evaluating the Grassmann integral $\int \mathcal{D}(\bar{\Psi}, \Psi)(\cdot)$ on the r.h.s. of (5.3).

The last second order representability condition which can be derived by the described method is the (optimal) G-Condition. Deriving this condition by Grassmann integration requires a choice of $\eta$, that is not as obvious as before. In the following, $\operatorname{tr}_{1}(\cdot)$ denotes the trace on $\mathcal{H}$ and $\operatorname{tr}_{2}(\cdot)$ the trace on $\mathcal{H} \otimes \mathcal{H}$.

Lemma 5.10. Theorem 4.18 implies the G-Condition:

$$
\forall A \in \mathcal{B}(\mathcal{H}): \operatorname{tr}_{2}\left(\left(A^{*} \otimes A\right)\left(\Gamma_{\vartheta}+\operatorname{Ex}\left(\gamma_{\vartheta} \otimes \mathbb{1}\right)\right)\right) \geq\left|\operatorname{tr}_{1}\left(A \gamma_{\vartheta}\right)\right|^{2}
$$

Proof. This time, we choose $\eta:=\left(\sum_{k, l \in M} \alpha_{k l} \bar{\psi}_{k} \star \psi_{l}-\beta\right) \star \vartheta$, where $\alpha_{k l} \in$ $\mathbb{C} \forall k, l \in M$ and $\beta:=\sum_{k, l \in M} \alpha_{k l} \int \mathcal{D}(\bar{\Psi}, \Psi) \vartheta^{*} \star \vartheta \star \bar{\psi}_{k} \star \psi_{l}$. Before we apply 
Theorem 4.18, we emphasize that by the CAR

$$
\begin{aligned}
& \left(\sum_{k, l \in M} \alpha_{k l} \bar{\psi}_{k} \psi_{l}-\beta\right)^{*} \star\left(\sum_{k, l \in M} \alpha_{k l} \bar{\psi}_{k} \psi_{l}-\beta\right) \\
= & \bar{\beta} \beta-\beta \sum_{k, l \in M} \bar{\alpha}_{k l} \bar{\psi}_{l} \star \psi_{k}-\bar{\beta} \sum_{m, n \in M} \alpha_{m n} \bar{\psi}_{m} \star \psi_{n} \\
& -\sum_{k, l \in M} \bar{\alpha}_{k l} \alpha_{m n} \bar{\psi}_{l} \star \bar{\psi}_{m} \star \psi_{k} \star \psi_{n}+\sum_{k, l, n \in M} \bar{\alpha}_{k l} \alpha_{k n} \bar{\psi}_{l} \star \psi_{n} .
\end{aligned}
$$

We consider the last two lines separately and integrate. The integration of the line before the last line in Eq. (5.4) yields

$$
\begin{aligned}
& \int \mathcal{D}(\bar{\Psi}, \Psi) \vartheta^{*} \star \vartheta \star\left(\bar{\beta} \beta-\beta \sum_{k, l \in M} \bar{\alpha}_{k l} \bar{\psi}_{l} \star \psi_{k}-\bar{\beta} \sum_{m, n \in M} \alpha_{m n} \bar{\psi}_{m} \star \psi_{n}\right) \\
= & \bar{\beta} \beta-\beta \bar{\beta}-\bar{\beta} \beta=-\bar{\beta} \beta,
\end{aligned}
$$

which follows from the definition of $\beta$. It is important to notice that $\beta$ does not depend on $\psi$ or $\bar{\psi}$ and, therefore, is a constant with respect to the Grassmann integration. In detail, we have for $\beta$

$$
\beta=\sum_{k, l \in M} \alpha_{k l} \int \mathcal{D}(\bar{\Psi}, \Psi) \vartheta^{*} \star \vartheta \star \bar{\psi}_{k} \star \psi_{l}=\operatorname{tr}_{1}\left(A \gamma_{\vartheta}\right),
$$

if we set $\left\langle\psi_{k}, A \psi_{l}\right\rangle:=\alpha_{k l}$ for every $k, l \in M$ and $A \in \mathcal{B}(\mathcal{H})$. The evaluation of the Grassmann integral of the last line in Eq. (5.4) provides

$$
\begin{aligned}
& -\sum_{k, l \in M} \bar{\alpha}_{k l} \alpha_{m n} \int \mathcal{D}(\bar{\Psi}, \Psi) \vartheta^{*} \star \vartheta \star \bar{\psi}_{l} \star \bar{\psi}_{m} \star \psi_{k} \star \psi_{n} \\
& +\sum_{k, l, n \in M} \bar{\alpha}_{k l} \alpha_{k n} \int \mathcal{D}(\bar{\Psi}, \Psi) \vartheta^{*} \star \vartheta \star \bar{\psi}_{l} \star \psi_{n} \\
= & \operatorname{tr}_{2}\left(\left(A^{*} \otimes A\right)\left(\Gamma_{\vartheta}+\operatorname{Ex}\left(\gamma_{\vartheta} \otimes \mathbb{1}\right)\right)\right) .
\end{aligned}
$$

Summing up, calculation (5.5) together with Eqs. (5.6) and (5.7) gives

$$
\operatorname{tr}_{2}\left(\left(A^{*} \otimes A\right)\left(\Gamma_{\vartheta}+\operatorname{Ex}\left(\gamma_{\vartheta} \otimes \mathbb{1}\right)\right)\right)-\left|\operatorname{tr}_{1}\left(A \gamma_{\vartheta}\right)\right|^{2} \geq 0,
$$

due to Theorem 4.18.

We summarize our results using $\mathcal{G}_{M}^{(2)}$ : 
Theorem 5.11. Let $\vartheta \star \vartheta^{*}$ be a Grassmann density, $\gamma_{\vartheta}$ its 1-pdm, and $\Gamma_{\vartheta}$ its 2-pdm. Then the following statements are equivalent:

(a) $\left(\gamma_{\vartheta}, \Gamma_{\vartheta}\right)$ fulfills $0 \leq \gamma_{\vartheta} \leq \mathbb{1}$ and the $G$-, $P$-, and Q-Conditions.

(b) $\forall \mu \in \mathcal{G}_{M}^{(2)}: \int \mathcal{D}(\bar{\Psi}, \Psi) \vartheta^{*} \star \vartheta \star \mu \geq 0$.

Proof. Again, we use Theorem 3.1 of [2] and the bijection property of $\Theta$, which ensures that the space of polynomials of degree lower or equal than four in creation and annihilation operators is mapped one-to-one to $\mathcal{G}_{M}^{(2)}$.

\section{3. $\mathbf{T}_{1-}$ and generalized $\mathbf{T}_{2}$-Condition}

The previous sections imply that further conditions on $\gamma_{\vartheta}$ and $\Gamma_{\vartheta}$ can be found by taking into account monomials of higher order of the form $\bar{\psi}_{i_{1}} \star$ $\cdots \star \bar{\psi}_{i_{n}} \star \psi_{j_{1}} \star \cdots \star \psi_{j_{n}}$ for $n>2$. Here we face the problem that monomials with $n>2$ have to "decompose" into monomials with $n \leq 2$. Due to this, only some choices of higher order monomials are suitable to derive further representability conditions. One of such monomials is given by

$$
\tau_{1}:=\sum_{i, j, k \in M} T_{i j k} \psi_{i} \star \psi_{j} \star \psi_{k} \in \mathcal{G}_{M}
$$

where, due to $\left\{\psi_{i}, \psi_{j}\right\}_{\star}=0, T_{i j k} \in \mathbb{C}$ is totally antisymmetric, i. e., $T_{i j k}=$ $-T_{j i k}=T_{j k i}$. The $\mathrm{T}_{1}$-Condition is the following.

Theorem 5.12. Let $T_{q} \in \mathcal{B}(\mathcal{H})$ be trace class. Set $T_{k q n}:=\left[T_{q}\right]_{k n}$ and $F_{T_{q}}:=$ $\sum_{k, n \in M} \bar{T}_{k q n}\left(\varphi_{k} \otimes \varphi_{n}\right) \in \mathcal{H} \otimes \mathcal{H}$. Then Theorem 4.18 implies the $\mathrm{T}_{1}$-Condition

$$
\sum_{q \in M}\left(2 \operatorname{tr}_{1}\left(\left|T_{q}\right|^{2}\right)-6 \operatorname{tr}_{1}\left(\left|T_{q}\right|^{2} \gamma_{\vartheta}\right)+3\left\langle F_{T_{q}}, \Gamma_{\vartheta} F_{T_{q}}\right\rangle\right) \geq 0
$$

Proof. We begin by considering the anticommutator $\left\{\tau_{1}^{*}, \tau_{1}\right\}_{\star} \in \mathcal{G}_{M}$ and observe that, by construction, $\left\{\tau_{1}^{*}, \tau_{1}\right\}_{\star} \geq 0$. Furthermore, we can use the CAR to establish normal-order in $\left\{\tau_{1}^{*}, \tau_{1}\right\}_{\star}$. The $(i, j)$-th matrix element of $A \in \mathcal{B}(\mathcal{H})$ is denoted by $[A]_{i j}:=\left\langle\psi_{i}, A \psi_{j}\right\rangle$. Using the antisymmetry of $T_{i j k}$ 
we obtain

$$
\begin{aligned}
\left\{\tau_{1}^{*}, \tau_{1}\right\}_{\star}= & 9 \sum_{l \in M} \sum_{i, j, m, n \in M} \bar{T}_{l j m} T_{l i n} \bar{\psi}_{m} \star \bar{\psi}_{j} \star \psi_{i} \star \psi_{n} \\
& +18 \sum_{m, l \in M} \sum_{k, n \in M} \bar{T}_{k m l} T_{l m n} \bar{\psi}_{k} \star \psi_{n}+6 \sum_{l, m, n \in M} \bar{T}_{l m n} T_{l m n} \\
= & 9 \sum_{q \in M} \sum_{i, j, m, n \in M}\left[T_{q}^{*}\right]_{m j}\left[T_{q}\right]_{i n} \bar{\psi}_{m} \star \bar{\psi}_{j} \star \psi_{i} \star \psi_{n} \\
& -18 \sum_{q \in M} \sum_{k, n \in M}\left[T_{q}^{*} T_{q}\right]_{k n} \bar{\psi}_{k} \star \psi_{n}+6 \sum_{q \in M} \operatorname{tr}_{1}\left(\left|T_{q}\right|^{2}\right) .
\end{aligned}
$$

Since $\left\{\tau_{1}^{*}, \tau_{1}\right\}_{\star} \geq 0$, we have by Theorem 4.18

$$
\int \mathcal{D}(\bar{\Psi}, \Psi) \vartheta \star\left\{\tau_{1}^{*}, \tau_{1}\right\}_{\star} \star \vartheta^{*} \geq 0 .
$$

Together with Eq. (5.2), the latter calculations and this positivity of the integral bring us to

$$
\begin{aligned}
0 \leq & 3 \sum_{q \in M} \sum_{i, j, m, n \in M}\left[T_{q}^{*}\right]_{m j}\left[T_{q}\right]_{i n}\left\langle\psi_{i} \otimes \psi_{n}, \Gamma_{\vartheta}\left(\psi_{j} \otimes \psi_{m}\right)\right\rangle \\
& -6 \sum_{q \in M} \sum_{k, n \in M}\left[\left|T_{q}\right|^{2}\right]_{k n}\left\langle\psi_{n}, \gamma_{\vartheta} \psi_{k}\right\rangle+2 \sum_{q \in M} \operatorname{tr}_{1}\left(\left|T_{q}\right|^{2}\right) .
\end{aligned}
$$

With $\left\langle\psi_{i}, T_{q} \psi_{j}\right\rangle=:\left[T_{q}\right]_{i j}$ and $F_{T_{q}}:=\sum_{k, n \in M} \bar{T}_{k q n}\left(\varphi_{k} \otimes \varphi_{n}\right)$, this yields the assertion.

The generalized $\mathrm{T}_{2}$-Condition can be derived equivalently by another choice of $\tau$. Using the anticommutator with a combination of two $\bar{\psi}$ 's and one $\psi$ (or vice versa), we have three different possibilities: $\tau_{2 a}:=\sum_{i, j, k \in M} T_{i j k}^{(a)} \bar{\psi}_{i} \star$ $\bar{\psi}_{j} \star \psi_{k}, \tau_{2 b}:=\sum_{i, j, k \in M} T_{i j k}^{(b)} \bar{\psi}_{i} \star \psi_{j} \star \bar{\psi}_{k}$, and $\tau_{2 c}:=\sum_{i, j, k \in M} T_{i j k}^{(c)} \psi_{i} \star \bar{\psi}_{j} \star \bar{\psi}_{k} . \mathrm{A}$ generalization of these possibilities is given by

$$
\tau_{2}:=\sum_{i, j, k \in M} T_{i j k} \bar{\psi}_{i} \star \bar{\psi}_{j} \star \psi_{k}+\sum_{i \in M} a_{i} \bar{\psi}_{i},
$$

where $T_{i j k}, a_{i} \in \mathbb{C} \forall i, j, k \in M$. This is a generalization, since we obtain $\tau_{2}=\tau_{2 a}$ for $a_{i} \equiv 0$ and $T_{i j k} \equiv T_{i j k}^{(a)}, \tau_{2}=\tau_{2 b}$ for $a_{i}=\sum_{j \in M} T_{i j j}^{(b)}$ and $T_{i j k}=$ 
$-T_{i k j}^{(b)}$, and, finally, $\tau_{2}=\tau_{2 c}$ for $a_{i}=\sum_{j \in M}\left(T_{j j i}^{(c)}-T_{j i j}^{(c)}\right)$ and $T_{i j k}=T_{k i j}^{(c)}$. The identities can be seen using the CAR. Unfortunately, if one uses the generalization $\tau_{2}$, symmetry properties on $T_{i j k}$ like, for example, $T_{i j k}^{(a)}=-T_{j i k}^{(a)}$ in $\tau_{2 a}$ or $T_{i j k}^{(c)}=-T_{i k j}^{(c)}$ in $\tau_{2 c}$ vanish. The generalized $\mathrm{T}_{2}$-Condition arises from $\left\{\tau_{2}^{*}, \tau_{2}\right\}_{\star} \geq 0$. In order to state the condition in a compact form, we need some new notation.

Definition 5.13. For $T_{k} \in \mathcal{B}(\mathcal{H}),\left[T_{k}\right]_{i j}:=T_{i j k} \forall i, j, k \in M$, and $\underline{a} \in \mathbb{C}^{|M|}$, we define $G_{T_{k}} \in \mathcal{H} \otimes \mathcal{H}$ and the matrices $Q_{1} \in \mathcal{B}(\mathcal{H} \otimes \mathcal{H})$ and $Q_{2}, Q_{3} \in$ $\mathcal{B}(\mathcal{H})$ by

$$
\begin{aligned}
& G_{T_{k}}:=\sum_{i, j \in M}\left[T_{k}\right]_{i j}\left(\psi_{i} \otimes \psi_{j}\right), \\
& \left\langle\psi_{k} \otimes \psi_{m}, Q_{1}\left(\psi_{n} \otimes \psi_{j}\right)\right\rangle:=\left[\bar{T}_{k}^{(A)} T_{n}^{(A)}\right]_{j m}, \\
& \left\langle\psi_{i}, Q_{2} \psi_{j}\right\rangle:=\operatorname{tr}_{1}\left(\left(T_{i}^{(A)}\right)^{*} T_{j}\right), \\
& \left\langle\psi_{i}, Q_{3} \psi_{j}\right\rangle:=\sum_{q \in M}\left(\left[\left(T_{i}^{(A)}\right)^{*}\right]_{j q} a_{q}+\left[T_{j}^{(A)}\right]_{i q} \bar{a}_{q}\right),
\end{aligned}
$$

where $\left[T_{k}^{(A)}\right]_{i j}:=\frac{1}{2}\left(\left[T_{k}\right]_{i j}-\left[T_{k}\right]_{j i}\right)=-\left[T_{k}^{(A)}\right]_{j i}$ denotes the antisymmetric part of $T_{k}$.

Theorem 5.14. Let $T_{k}, \underline{a}, G_{T_{q}}$ and $Q_{1}, Q_{2}, Q_{3}$ be as in Definition 5.13. Then Theorem 4.18 implies the generalized $\mathrm{T}_{2}$-Condition

$$
\sum_{q \in M}\left\langle G_{T_{q}}, \Gamma_{\vartheta} G_{T_{q}}\right\rangle+4 \operatorname{tr}_{2}\left(Q_{1} \Gamma_{\vartheta}\right)+2 \operatorname{tr}_{1}\left(\left(Q_{2}+Q_{3}\right) \gamma_{\vartheta}\right)+|\underline{a}|^{2} \geq 0 .
$$

Proof. The first task is to bring $\left\{\tau_{2}^{*}, \tau_{2}\right\}$ into normal-order. Then the two terms of third order cancel and only terms of order less than or equal to two remain. To calculate the anticommutator we use $\left\{(\mu+\eta)^{*}, \mu+\eta\right\}_{\star}=$ $\left\{\mu^{*}, \mu\right\}_{\star}+2 \mathfrak{R e}\left\{\mu^{*}, \eta\right\}_{\star}+\left\{\eta^{*}, \eta\right\}_{\star}$ for $\mu:=\sum_{i, j, k \in M} T_{i j k} \bar{\psi}_{i} \star \bar{\psi}_{j} \star \psi_{k}$ and $\eta:=$ $\sum_{i \in M} a_{i} \bar{\psi}_{i}$. Here, $\mathfrak{R e}$ denotes the real part. By the CAR, we have

$$
\left\{\eta^{*}, \eta\right\}_{\star}=\sum_{i \in M}\left|a_{i}\right|^{2}, \quad\left\{\mu^{*}, \eta\right\}_{\star}=\sum_{k, n \in M} \sum_{q \in M}\left(\bar{T}_{q n k}-\bar{T}_{n q k}\right) a_{q} \bar{\psi}_{k} \star \psi_{n},
$$


and

$$
\begin{aligned}
\left\{\mu^{*}, \mu\right\}_{\star}= & \left(\sum_{j, k, m, n \in M} \sum_{q \in M}\left(\left(\bar{T}_{j q k}-\bar{T}_{q j k}\right)\left(T_{q m n}-T_{m q n}\right)+\bar{T}_{n j q} T_{k m q}\right)\right. \\
& \left.\times \bar{\psi}_{k} \star \bar{\psi}_{m} \star \psi_{j} \star \psi_{n}\right)+\sum_{k, n \in M} \sum_{p, q \in M}\left(\bar{T}_{p q k}-\bar{T}_{q p k}\right) T_{p q n} \bar{\psi}_{k} \star \psi_{n} .
\end{aligned}
$$

We set $T_{i j q}=:\left[T_{q}\right]_{i j}$ where $T_{q} \in \mathcal{B}(\mathcal{H}) \forall q \in M$ and observe that $\left[\bar{T}_{q}\right]_{i j}=$ $\left[T_{q}^{*}\right]_{j i}, \bar{T}_{q n k}-\bar{T}_{n q k}=2\left[\left(T_{k}^{(A)}\right)^{*}\right]_{n q}$, and $T_{q m n}-T_{m q n}=2\left[T_{n}^{(A)}\right]_{q m}$, where $T^{(A)}$ is the antisymmetric part of $T$ (see Definition 5.13). This allows us to rewrite the anticommutators as

$$
2 \mathfrak{R e}\left\{\mu^{*}, \eta\right\}_{\star}=2 \sum_{k, n \in M} \sum_{q \in M}\left(\left[\left(T_{k}^{(A)}\right)^{*}\right]_{n q} a_{q}+\left[T_{n}^{(A)}\right]_{q k} \bar{a}_{q}\right) \bar{\psi}_{k} \star \psi_{n}
$$

and

$$
\begin{aligned}
\left\{\mu^{*}, \mu\right\}_{\star}= & \left(\sum_{j, k, m, n \in M} \sum_{q \in M}\left(4\left[\left(T_{k}^{(A)}\right)^{*}\right]_{q j}\left[T_{n}^{(A)}\right]_{q m}+\left[T_{q}^{*}\right]_{j n}\left[T_{q}\right]_{k m}\right)\right. \\
& \left.\times \bar{\psi}_{k} \star \bar{\psi}_{m} \star \psi_{j} \star \psi_{n}\right)+2 \sum_{k, n \in M} \sum_{p, q \in M}\left[\left(T_{k}^{(A)}\right)^{*}\right]_{q p}\left[T_{n}\right]_{p q} \bar{\psi}_{k} \star \psi_{n} .
\end{aligned}
$$

In the next step we use $\left\langle\psi_{i}, A \psi_{j}\right\rangle=[A]_{i j}$ for $A \in \mathcal{B}(\mathcal{H})$ and the Grassmann representation of $\gamma$ and $\Gamma$ from Eqs. (5.1) and (5.2). Definition 5.13 then leads to

$$
\begin{aligned}
& \sum_{j, k, m, n \in M} \sum_{q \in M}\left[T_{q}^{*}\right]_{j n}\left[T_{q}\right]_{k m} \int \mathcal{D}(\bar{\Psi}, \Psi) \vartheta^{*} \star \vartheta \star \bar{\psi}_{k} \star \bar{\psi}_{m} \star \psi_{j} \star \psi_{n} \\
= & \sum_{q \in M}\left\langle G_{T_{q}}, \Gamma_{\vartheta} G_{T_{q}}\right\rangle
\end{aligned}
$$

for $G_{T_{q}}:=\sum_{i, j \in M}\left[T_{q}\right]_{i j}\left(\psi_{i} \otimes \psi_{j}\right) \in \mathcal{H} \otimes \mathcal{H}$. With $\left\langle\psi_{m} \otimes \psi_{k}, Q_{1}\left(\psi_{j} \otimes \psi_{n}\right)\right\rangle:=$ $\left[\bar{T}_{k}^{(A)} T_{n}^{(A)}\right]_{j m}$ we have

$$
\begin{aligned}
& 4 \sum_{\substack{j, k, m, n, q \in M \\
=}}\left[\bar{T}_{k}^{(A)}\right]_{j q}\left[T_{n}^{(A)}\right]_{q m} \int \mathcal{D}(\bar{\Psi}, \Psi) \vartheta^{*} \star \vartheta \star \bar{\psi}_{k} \star \bar{\psi}_{m} \star \psi_{j} \star \psi_{n} \\
= & 4 \operatorname{tr}_{2}\left(Q_{1} \Gamma_{\vartheta}\right) .
\end{aligned}
$$


Furthermore,

$$
2 \sum_{k, n \in M} \sum_{p, q \in M}\left[\left(T_{k}^{(A)}\right)^{*}\right]_{q p}\left[T_{n}\right]_{p q} \int \mathcal{D}(\bar{\Psi}, \Psi) \vartheta^{*} \star \vartheta \star \bar{\psi}_{k} \star \psi_{n}=2 \operatorname{tr}_{1}\left(Q_{2} \gamma_{\vartheta}\right)
$$

for $\left[Q_{2}\right]_{k n}:=\operatorname{tr}_{1}\left(\left(T_{k}^{(A)}\right)^{*} T_{n}\right)$. Finally, we have

$$
2 \mathfrak{R e} \int \mathcal{D}(\bar{\Psi}, \Psi) \vartheta^{*} \star \vartheta \star\left\{\mu^{*}, \eta\right\}_{\star}=2 \operatorname{tr}_{1}\left(Q_{3} \gamma_{\vartheta}\right)
$$

where $\left[Q_{3}\right]_{i j}:=\sum_{q \in M}\left(\left[\left(T_{i}^{(A)}\right)^{*}\right]_{j q} a_{q}+\left[T_{j}^{(A)}\right]_{q i} \bar{a}_{q}\right) \cdot \sum_{i}\left|a_{i}\right|^{2}=:|\underline{a}|^{2}$ is the squared unitary norm of $\underline{a}$. The proof is complete by inserting the latter calculations into the inequality of Theorem 4.18.

As already mentioned, we have antisymmetry properties for certain choices of $\underline{a}$ and $T_{i j k}$. In $\tau_{2 a}$, which we gain by setting $\underline{a} \equiv 0$ and $T_{i j k}=T_{i j k}^{(a)}=$ $\left[T_{k}^{(a)}\right]_{i j}$, we have $\left[T_{k}\right]_{i j}=-\left[T_{k}\right]_{j i}$ or $T_{k} \equiv T_{k}^{(A)}$. In this case, we have a simplification of the generalized $\mathrm{T}_{2}$-Condition:

Corollary 5.15. For $\underline{a} \equiv 0, T_{k} \equiv T_{k}^{(A)},\left[\widetilde{T}_{k}\right]_{i j}:=\left[T_{j}\right]_{i k}$, we have the $\mathrm{T}_{2 a^{-}}$ Condition given by

$$
\sum_{q \in M}\left(\left\langle G_{\widetilde{T}_{q}}, \Gamma_{\vartheta} G_{\widetilde{T}_{q}}\right\rangle+4 \operatorname{tr}_{2}\left(\left(\widetilde{T}_{q}^{*} \otimes \widetilde{T}_{q}\right) \Gamma_{\vartheta}\right)+2 \operatorname{tr}_{1}\left(\left|\widetilde{T}_{q}\right|^{2} \gamma_{\vartheta}\right)\right) \geq 0
$$

We can also use an antisymmetry property in $\tau_{2 c}$ which leads to a condition $\mathrm{T}_{2 c}$. Unfortunately, there is no simplification compared to the generalized $\mathrm{T}_{2}$-Condition. There is, however, no antisymmetry property in $\tau_{2 b}$.

Since $\left\{\tau_{1}^{*}, \tau_{1}\right\}_{\star},\left\{\tau_{2}^{*}, \tau_{2}\right\}_{\star} \in \mathcal{G}_{M}^{(3)}$, the $\mathrm{T}_{1}$ - and $\mathrm{T}_{2}$-Conditions are conditions of third order.

\section{Quasifree Grassmann states}

The notion of Grassmann integration allows for a calculation of traces on the fermion Fock space by Grassmann integrals and, in turn, to reformulate representability condition in terms of Grassmann integrals. At last, we consider quasifree states, their one-particle density matrices, and the expression of their relation in terms of Grassmann integrals. 
In the following, we will abbreviate the expectation value of a Grassmann variable $\mu \in \mathcal{G}_{M}$ with respect to a Grassmann density $\varkappa \in \mathcal{G}_{M}$ by

$$
\int \mathcal{D}(\bar{\Psi}, \Psi) \varkappa \star \mu=:\langle\mu\rangle_{\varkappa}
$$

Definition 6.1. Let $\left\{\bar{\psi}_{i}, \psi_{i}\right\}_{i \in M}$ be a set of generators of $\mathcal{G}_{M}$ and let $\widetilde{\psi}_{i}$ denote either $\psi_{i} \in \mathcal{G}_{M}$ or $\bar{\psi}_{i} \in \mathcal{G}_{M}$. We call a Grassmann density $\varkappa$ quasifree if

(i) $\left\langle\widetilde{\psi}_{1} \star \widetilde{\psi}_{2} \star \cdots \star \widetilde{\psi}_{2 N-1}\right\rangle_{\varkappa}=0$ and

(ii) $\left\langle\widetilde{\psi}_{1} \star \widetilde{\psi}_{2} \star \cdots \star \widetilde{\psi}_{2 N}\right\rangle_{\varkappa}=\sum_{\pi}^{\prime}(-1)^{\pi}\left\langle\widetilde{\psi}_{\pi(1)} \star \widetilde{\psi}_{\pi(2)}\right\rangle_{\varkappa} \times \cdots$

$$
\times\left\langle\widetilde{\psi}_{\pi(2 N-1)} \star \widetilde{\psi}_{\pi(2 N)}\right\rangle_{\varkappa}
$$

for every $N \in \mathbb{N}$. Here $\sum_{\pi}^{\prime}$ denotes the sum over all permutations $\pi$ obeying $\pi(1)<\pi(3)<\cdots<\pi(2 N-1)$ and $\pi(2 j-1)<\pi(2 j)$ for all $1 \leq j \leq N$. The maximal number of (distinct) $\psi_{i}$ or $\bar{\psi}_{i}$ in (i) and (ii) is $|M|$.

Remark 6.2. We have to restrict $N$ in the latter definition or extend $M$ sufficiently, since the expression on the 1.h.s. of condition (i) and (ii), respectively, vanishes, if the number of $\psi_{i}$ or $\bar{\psi}_{i}$ is larger than $|M|$.

As it is known from [3], there is a unique characterization of quasifree states by the 1-pdm. In detail, assuming particle number-conservation and defining

$$
\widetilde{\gamma}:=\left(\begin{array}{cc}
\gamma & 0 \\
0 & \mathbb{1}-\bar{\gamma}
\end{array}\right) \in \mathcal{B}(\mathcal{H} \oplus \mathcal{H}),
$$

which is the generalized 1-pdm corresponding to $\gamma$, one has the following:

Theorem 6.3. Let $\widetilde{\gamma}=\left(\begin{array}{cc}\gamma & 0 \\ 0 & \mathbb{1}-\bar{\gamma}\end{array}\right)$ be an operator on $\mathcal{H} \oplus \mathcal{H}$ with $\operatorname{tr}_{1}(\gamma)<\infty$ and $0 \leq \widetilde{\gamma} \leq \mathbb{1}$. Then there is a unique quasifree state $\rho$ with $\operatorname{tr}_{\wedge \mathcal{H}}(\rho \widehat{\mathbb{N}})<\infty$ such that $\widetilde{\gamma}=\widetilde{\gamma}_{\rho}$.

For a proof see [3].

In the language of Grassmann integration, the reverse direction, namely that $\widetilde{\gamma}_{\varkappa}$, i. e., the generalized 1-pdm of a quasifree Grassmann density $\varkappa$, 
has to fulfill $0 \leq \widetilde{\gamma}_{\varkappa} \leq \mathbb{1}$, can be deduced by appropriate choices of $\phi \in \mathcal{G}_{M}$ in the positivity condition

$$
\left\langle\phi^{*} \star \phi\right\rangle_{\varkappa} \geq 0
$$

The aim of this section is to determine the unique quasifree Grassmann density subject to Theorem 6.3, i. e., the element of a Grassmann algebra corresponding the state given in [3]. To this end, we consider an operator $\widetilde{\gamma} \in \mathcal{B}(\mathcal{H} \oplus \mathcal{H})$ with $0 \leq \widetilde{\gamma} \leq \mathbb{1}$ and its eigenvalues $\lambda_{i}$ and $\left(1-\lambda_{i}\right)$, where $0 \leq \lambda_{i} \leq \frac{1}{2}, i \in M$. Furthermore, we define $P_{0}$ to be the projection onto the subspace of $\wedge \mathcal{H}$, on which $\sum_{i: \lambda_{i}=0} c_{i}^{*} c_{i}=0$ for $i \in M$. Moreover, for any $i \in M$ with $\lambda_{i} \neq 0$ the quantity $q_{i}$ is given by the relation $\left(1+\mathrm{e}^{q_{i}}\right)^{-1}=\lambda_{i}$. Then, according to [3], any operator $\widetilde{\gamma}$ with $0 \leq \widetilde{\gamma} \leq \mathbb{1}$ is the generalized 1 -pdm of a unique quasifree state $\rho \in \mathcal{B}(\wedge \mathcal{H})$ given by

$$
\rho:=\frac{G}{\operatorname{tr}_{\wedge \mathcal{H}}(G)},
$$

where

$$
G:=P_{0} \mathrm{e}^{-H} \quad \text { and } \quad H:=\sum_{i: \lambda_{i} \neq 0} q_{i} c_{i}^{*} c_{i} .
$$

Before we turn to the definition of the Grassmann density corresponding to (6.1), we introduce the abbreviations $\Theta_{0}:=\Theta\left(P_{0}\right) \in \mathcal{G}_{M}$ and $\prod_{i=1}^{n \star} \mu_{i}:=$ $\mu_{1} \star \mu_{2} \star \cdots \star \mu_{n}$ for $\mu_{1}, \ldots, \mu_{n} \in \mathcal{G}_{M}, n \in \mathbb{N}$. Furthermore, we associate the generators $\left\{\bar{\psi}_{i}, \psi_{i}\right\}_{i \in M}$ of $\mathcal{G}_{M}$ with the ONB $\left\{\psi_{i}\right\}_{i \in M}$ of $\mathcal{H}$, where the $\psi_{i}$ are the eigenvectors of $\gamma$ corresponding to the eigenvalues $\lambda_{i}$ and $\left(1-\lambda_{i}\right)$.

Lemma 6.4. Let $\left\{\psi_{i}\right\}_{i \in M}$ be an $O N B$ of $\mathcal{H}$ such that $\gamma \psi_{i}=\lambda_{i} \psi_{i}$ and let $\mathcal{G}_{M}$ be generated by $\left\{\bar{\psi}_{i}, \psi_{i}\right\}_{i \in M}$. The Grassmann density $\varkappa \in \mathcal{G}_{M}$ corresponding to $\rho=\frac{G}{\operatorname{tr}_{\wedge \mathcal{H}}(G)}$ is given by

$$
\varkappa=\frac{1}{Z}\left(\Theta_{0} \star \prod_{i: \lambda_{i} \neq 0}^{\star}\left(\left(\mathrm{e}^{-q_{i}}-1\right) \bar{\psi}_{i} \psi_{i}+1\right)\right),
$$

where

$$
Z:=\int \mathcal{D}(\bar{\Psi}, \Psi) \Theta_{0} \star \prod_{i: \lambda_{i} \neq 0}^{\star}\left(\left(\mathrm{e}^{-q_{i}}-1\right) \bar{\psi}_{i} \psi_{i}+1\right)
$$


Proof. We consider $\Theta(\rho)$ with $\rho$ as in (6.1). First, we observe that $c_{i}^{*} c_{i}$ commutes with $c_{k}^{*} c_{k}$ for every $i, k$. Therefore, we have

$$
\mathrm{e}^{-H}=\prod_{i: \lambda_{i} \neq 0}\left(\sum_{n=1}^{\infty} \frac{\left(-q_{i}\right)^{n}}{n !} c_{i}^{*} c_{i}+1\right)=\prod_{i: \lambda_{i} \neq 0}\left(\left(\mathrm{e}^{-q_{i}}-1\right) c_{i}^{*} c_{i}+1\right),
$$

since $\left(c_{i}^{*} c_{i}\right)^{n}=c_{i}^{*} c_{i}$. Thus,

$$
\begin{aligned}
\Theta\left(P_{0} \mathrm{e}^{-H}\right) & =\Theta_{0} \star \Theta\left(\prod_{i: \lambda_{i} \neq 0}\left(\left(\mathrm{e}^{-q_{i}}-1\right) c_{i}^{*} c_{i}+1\right)\right) \\
& =\Theta_{0} \star \prod_{i: \lambda_{i} \neq 0}^{\star}\left(\left(\mathrm{e}^{-q_{i}}-1\right) \bar{\psi}_{i} \psi_{i}+1\right),
\end{aligned}
$$

where we have used $\Theta(A B)=\Theta(A) \star \Theta(B)$.

The Grassmann state corresponding to the Grassmann density (6.2) is given by the map

$$
\mathcal{G}_{M} \rightarrow \mathbb{C}, \quad \mu \mapsto\langle\mu\rangle_{\varkappa}
$$

We want to check that the Grassmann density from Lemma 6.4 is quasifree, i. e., fulfills conditions (i) and (ii) from Definition 6.1. The uniqueness of $\varkappa$ follows from the bijection property of the map $\Theta$.

Theorem 6.5. The Grassmann density $\varkappa$ in Lemma 6.4 is quasifree.

Proof. We consider the Grassmann variable

$$
\varkappa_{\mu}:=\prod_{i \in M}^{\star}\left(r_{i} \bar{\psi}_{i} \psi_{i}+1\right)
$$

where $r_{i}:=\mathrm{e}^{-q_{i}(\mu)}-1$ and $q_{i}(\mu) \equiv \mu \in \mathbb{R}$ for all $i$ with $\lambda_{i}=0$ and $q_{i}(\mu) \equiv q_{i}$ for all $i$ with $\lambda_{i} \neq 0$. The quasifreeness of $\varkappa$ follows from the quasifreeness of $\varkappa_{\mu}$ by a limiting argument. The first claim of Definition 6.1 is immediate for $\varkappa_{\mu}$, since the Grassmann integral vanishes for any odd number of $\widetilde{\psi}$ 's. This can be seen by Remark 4.15 and the chequerboard. The validity of Equation (ii) of Definition 6.1 has already been proved in [10]. Here we emphasize the main steps and transfer the notation of [10] to Grassmann integrals. We 
consider the 1. h. s. of claim (ii) of Definition 6.1,

$$
\left\langle\widetilde{\psi}_{a} \star \widetilde{\psi}_{b} \star \widetilde{\psi}_{c} \star \cdots \star \widetilde{\psi}_{f}\right\rangle_{\varkappa_{\mu}}=\int \mathcal{D}(\bar{\Psi}, \Psi) \varkappa_{\mu} \star \widetilde{\psi}_{a} \star \widetilde{\psi}_{b} \star \widetilde{\psi}_{c} \star \cdots \star \widetilde{\psi}_{f}
$$

with $2 N$ generators $\widetilde{\psi}_{a}, \cdots, \widetilde{\psi}_{f}$. In the first step we eliminate $\widetilde{\psi}_{a}$ from the expectation value by a pull through formula. To this end we use $\left\{\widetilde{\psi}_{a}, \widetilde{\psi}_{b}\right\}_{\star}:=$ $\widetilde{\psi}_{a} \star \widetilde{\psi}_{b}+\widetilde{\psi}_{b} \star \widetilde{\psi}_{a}$, which is either $1,-1$, or 0 . This yields

$$
\begin{aligned}
& \left.\left\langle\widetilde{\psi}_{a} \star \widetilde{\psi}_{b} \star \widetilde{\psi}_{c} \star \cdots \star \widetilde{\psi}_{f}\right\rangle_{\varkappa_{\mu}} \tau_{=} \tilde{\psi}_{a}, \widetilde{\psi}_{b}\right\}_{\star}\left\langle\widetilde{\psi}_{c} \star \widetilde{\psi}_{d} \star \cdots \star \widetilde{\psi}_{f}\right\rangle_{\varkappa_{\mu}}-\left\{\widetilde{\psi}_{a}, \widetilde{\psi}_{c}\right\}_{\star}\left\langle\widetilde{\psi}_{b} \star \widetilde{\psi}_{d} \star \cdots \star \widetilde{\psi}_{f}\right\rangle_{\varkappa_{\mu}} \\
& +\left\{\widetilde{\psi}_{a}, \widetilde{\psi}_{d}\right\}_{\star}\left\langle\widetilde{\psi}_{b} \star \widetilde{\psi}_{c} \star \cdots \star \widetilde{\psi}_{f}\right\rangle_{\varkappa_{\mu}}+\cdots \\
& +\left\{\widetilde{\psi}_{a}, \widetilde{\psi}_{f}\right\}_{\star}\left\langle\widetilde{\psi}_{b} \star \widetilde{\psi}_{c} \star \cdots \star \widetilde{\psi}_{e}\right\rangle_{\varkappa_{\mu}}-\left\langle\widetilde{\psi}_{b} \star \widetilde{\psi}_{c} \star \cdots \star \widetilde{\psi}_{f} \star \widetilde{\psi}_{a}\right\rangle_{\varkappa_{\mu}} .
\end{aligned}
$$

Afterwards, we use the cyclicity of the Grassmann integral in the last expectation value on the r.h.s. of the latter expression and the identities

$$
\bar{\psi}_{i} \star \varkappa_{\mu}=\mathrm{e}^{q_{i}} \varkappa_{\mu} \star \bar{\psi}_{i} \text { and } \psi_{i} \star \varkappa_{\mu}=\mathrm{e}^{-q_{i}} \varkappa_{\mu} \star \psi_{i}
$$

which follow from the fact that $\varkappa_{\mu}$ is a star product of single states of the form $r_{i} \bar{\psi}_{i} \psi_{i}+1$ and the CAR for the star product. Thus, the last expectation value can be written as

$$
\left\langle\widetilde{\psi}_{b} \star \widetilde{\psi}_{c} \star \cdots \star \widetilde{\psi}_{f} \star \widetilde{\psi}_{a}\right\rangle_{\varkappa_{\mu}}=\mathrm{e}^{ \pm q_{a}}\left\langle\widetilde{\psi}_{a} \star \widetilde{\psi}_{b} \star \widetilde{\psi}_{c} \star \cdots \star \widetilde{\psi}_{f}\right\rangle_{\varkappa_{\mu}}
$$

and we conclude with

$$
\begin{aligned}
& \left\langle\widetilde{\psi}_{a} \star \widetilde{\psi}_{b} \star \widetilde{\psi}_{c} \star \cdots \star \widetilde{\psi}_{f}\right\rangle_{\varkappa_{\mu}}
\end{aligned}
$$

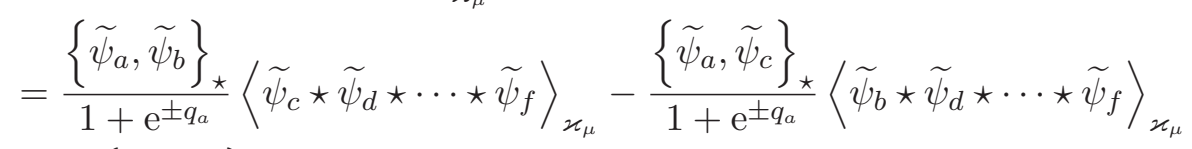

$$
\begin{aligned}
& +\frac{\left\{\widetilde{\psi}_{a}, \widetilde{\psi}_{d}\right\}_{\star}}{1+\mathrm{e}^{ \pm q_{a}}}\left\langle\widetilde{\psi}_{b} \star \widetilde{\psi}_{c} \star \cdots \star \widetilde{\psi}_{f}\right\rangle_{\varkappa_{\mu}}+\cdots \\
& +\frac{\left\{\widetilde{\psi}_{a}, \widetilde{\psi}_{f}\right\}_{\star}}{1+\mathrm{e}^{ \pm q_{a}}}\left\langle\widetilde{\psi}_{b} \star \widetilde{\psi}_{c} \star \cdots \star \widetilde{\psi}_{e}\right\rangle_{\varkappa_{\mu}} .
\end{aligned}
$$


We have reduced the expectation value of $2 N$ generators to a sum of expectation values of 2(N-1) generators. As in [10], the assertion follows by an induction in the number of generators. Finally, the quasifreeness of $\varkappa$ follows from

$$
\varkappa=\lim _{\mu \rightarrow \infty} \frac{\varkappa_{\mu}}{\int \mathcal{D}(\bar{\Psi}, \Psi) \varkappa_{\mu}}
$$

which completes the proof.

Remark 6.6. Carrying out the $|M|$-fold star product in $\varkappa_{\mu}$, we find a more convenient form of $\varkappa_{\mu}$ :

$$
\varkappa_{\mu}=\sum_{Q \subseteq M}(-1)^{s_{Q}} \prod_{i \in Q} r_{i} \prod_{i \in Q} \bar{\psi}_{i} \prod_{i \in Q} \psi_{i}=\sum_{Q \subseteq M}(-1)^{s_{Q}} r_{Q} \bar{\Psi}_{Q} \Psi_{Q},
$$

where $s_{Q}:=\frac{1}{2}|Q|(|Q|-1), r_{Q}:=\prod_{i \in Q} r_{i}$. The sum runs over all ordered subsets $Q \subseteq M$.

\section{References}

[1] V. Bach, Error Bound for the Hartree-Fock Energy of Atoms and Molecules. Communications in Mathematical Physics, 147(3) (1992), $527-548$.

[2] V. Bach, H. K. Knörr and E. Menge. Fermion Correlation Inequalities Derived from $G$ - and P-Conditions. Documenta Mathematica, 17(14) (2012), 451-481.

[3] V. Bach, E. H. Lieb and J. P. Solovej, Generalized Hartree-Fock Theory and the Hubbard Model. Journal of Statistical Physics, 76(1-2) (1994), $3-89$.

[4] E. Cancès, G. Stoltz and M. Lewin, The electronic ground state energy problem: A new reduced density matrix approach. The Journal of Chemical Physics, 125 (2006), 064101.

[5] A. J. Coleman, Structure of Fermion Density Matrices. Reviews of Modern Physics, 35(3) (1963), 668-687.

[6] M. Combescure and D. Robert, Coherent States and Applications in Mathematical Physics. Series: Theoretical and Mathematical Physics, Springer-Verlag, 2012. 
[7] R. M. Erdahl, Representability. International Journal of Quantum Chemistry, 13(6) (1978), 697-718.

[8] J. Feldman, H. Knörrer and E. Trubowitz, Fermionic Functional Integrals and the Renormalization Group. Volume 16 of CRM Monograph Series, American Mathematical Society, 2002.

[9] C. Garrod and J. K. Percus, Reduction of the N-Particle Variational Problem. Journal of Mathematical Physics, 5(12) (1964), 1756-1776.

[10] M. Gaudin, Une démonstration simplifiée du théorème de Wick en méchanique statistique. Nuclear Physics, 15 (1960), 89-91.

[11] E. H. Lieb and W. Thirring, Bound for the Kinetic Energy of Fermions which Proves the Stability of Matter. Physical Review Letters, 35(11) (1975), 687-689; Errata 35 (1975), 1116.

[12] P.-O. Löwdin, Quantum Theory of Many-Particle Systems. I. Physical Interpretations by Means of Density Matrices, Natural Spin-Orbitals, and Convergence Problems in the Method of Configurational Interaction. Physical Review, 97(6) (1955), 1474-1489.

[13] D. A. Mazziotti, Variational minimization of atomic and molecular ground-state energies via the two-particle reduced density matrix. Physical Review A, 65 (2002), 062511.

[14] D. A. Mazziotti, Structure of Fermionic Density Matrices: Complete N-Representability Conditions. Physical Review Letters, 108 (2012), 263002.

[15] W. d. S. Pedra, Zur mathematischen Theorie der Fermiflüssigkeiten bei positiven Temperaturen. PhD thesis, Universität Leipzig, 2005.

[16] M. Salmhofer, Renormalization - An Introduction. Springer-Verlag, 1998.

[17] L. A. Takhtajan, Quantum Mechanics for Mathematicians. Volume 95 of Graduate Studies in Mathematics, American Mathematical Society, 2008.

[18] W. Thirring, Quantenmechanik großer Systeme. Volume 4 of Lehrbuch der Mathematischen Physik, Springer-Verlag, 2008.

[19] Z. Zhao, B. J. Braams, M. Fukuda, M. L. Overton and J. K. Percus, The reduced density matrix method for electronic structure calculations and the role of three-index representability conditions. Journal of Chemical Physics, 120 (2004), 2095. 
Technische Universität Braunschweig, Institut für Analysis und Algebra, Rebenring 31, 38106 Braunschweig, Germany

E-mail address: v.bach@tu-bs.de

Aalborg Universitet, Institut for Matematiske FaG

Fredric Bajers Vej 7G, 9220 Aalborg, Denmark

E-mail address: hanskk@math.aau.dk

Technische Universität Braunschweig, Institut für Analysis und Algebra, Rebenring 31, 38106 Braunschweig, Germany

E-mail address: e.menge@tu-bs.de 